\title{
High Resolution Time-to-digital Conversion Circuit for HgCdTe APD Detector at 77K
}

\section{zhang qi wen ( $\nabla 54822417 @ s i n a . c o m$ )}

Shanghai Institute of Technology Physics,Chinese Academy of Sicences

\section{Chen Honglei}

Shanghai Institute of Technology physics, chinese academy of sciences

\section{Ding Ruijun}

shanghai institute of technology physics, chinese academy of sciences

\section{Research Article}

Keywords: HgCdTe APD, time-to-digital converter circuit, Vernier delay line, Nonlinearity

Posted Date: February 8th, 2021

DOI: https://doi.org/10.21203/rs.3.rs-161259/v1

License: (1) This work is licensed under a Creative Commons Attribution 4.0 International License. Read Full License 


\title{
High resolution time-to-digital conversion circuit for $\mathrm{HgCdTe}$ APD detector at 77K
}

\author{
Zhang Qiwen ${ }^{1,2}$,Chen Honglei ${ }^{1}$,Ding Ruijun ${ }^{1 *}$ \\ (1. Key Laboratory of Infrared Imaging Materials and Detectors,Shanghai Institute of Technology \\ Physics,Chinese Academy of Sciences, Shanghai 200083,China; \\ 2. University of Chinese Academy of Sciences,Beijing 100049,China)
}

E-mail addresses:zhangqiwen1027@sina.com(QW Zhang),dingrj@mail.sitp.ac.cn(RJ Ding)

\begin{abstract}
HgCdTe avalanche photodiode (APD) is a frontier research on infrared focal plane technology, High-precision time stamp readout circuit is the basis of the APD focal plane at $77 \mathrm{~K}$, which directly affects APD infrared focal plane performance. Time-to-digital conversion circuit (TDC) is one of the methods to achieve high-precision time stamping. Based on the analysis of MOSFET at low temperature, our design a vernier TDC circuit, which uses a synchronous counter to quantize an integer multiple of the period to achieve a coarse count of 6 bits; The on-chip PLL multiplied high-frequency clock has high-precision and high PVT characteristics, using it to quantify the part that is less than one clock cycle to achieve a fine-count of 6 bits output. The circuit adopts standard CMOS process tape out, our circuit works at a master-frequency of $120 \mathrm{MHz}$. At $77 \mathrm{~K}$, the circuit test can distinguish the time resolution of 236.280 ps. The DNL is within -0.54 0.71 LSB, and INL is within -1.32 1.21 LSB.
\end{abstract}

Key words: HgCdTe APD; time-to-digital converter circuit; Vernier delay line; Nonlinearity

\section{Introduction}

The mercury cadmium telluride avalanche photodiode ( $\mathrm{HgCdTe}$ APD) detector is a new type of detector developed in recent years (A Roga lsk et al. 2009; Lu et al. 2011). Compared with other material detectors, it has very high gain in linear mode (J. Beck et al.), ranging from tens to hundreds of times. At the same time, it has low excessive noise and high sensitivity, which can achieve single photon detection; Moreover, in the active and passive mode, it can obtain the intensity information and time information of the target object simultaneously, to realize real-time three-dimensional detection.

In the three-dimensional imaging ranging, The HgCdTe APD readout circuit (E.Allen.2012) converts optical signals into electrical signals and then outputs it. the TDC (Luo 2014) in the analog-to-digital conversion is an important part of the readout circuit. It converts the analog signal carrying time information into a digital signal, achieve the quantification of time information, and its resolution will 
determine the minimum time that can be distinguished (Feng 2010).

At present, the reports on the focal plane $\mathrm{HgCdTe}$ APD readout circuit at low temperature are mainly concentrated in the French SOFRADIR company and US DRS company. For example, the French SOFRADIR company reported that the resolution of 100 ps can be achieved under the form of CTIA structure with two negative feedback capacitors at $77 \mathrm{~K}$ (Rothman, J et al. 2016). RAYTHEON company reported to achieve 300 ps resolution under $77 \mathrm{~K}$ conditions (Jack, Michael, et al. 2011); There are few domestic researches in this filed. Among them, Southeast University used the delay chain phase-locked loop structure to achieve $1 \mathrm{~ns}$ accuracy at room temperature. Because of the single delay chain, it is limited by the resolution of a single delay gate (Zheng et al. 2017).

Based on the advantages of $\mathrm{HgCdTe}$ APD working at $77 \mathrm{~K}$, study the TDC structure of the readout circuit at low temperature to achieve high-precision time resolution. Our design uses a vernier-type double delay line TDC structure, so that the delay resolution is not affected by the delay of a single gate, At the same time, the delay unit bias voltage is externally connected to achieve voltage selection. The tape-out uses the CSMC 0.5 um 2P3M process, and at $77 \mathrm{~K}$ the test results can distinguish the time resolution of $236.28 \mathrm{ps}$, power consumption is $14.459 \mathrm{~mW}$.

\section{Detector noise analysis}

The detector noise is mainly composed of three parts: 1/f noise, generation-composite noise, and white noise. This paper first analyzes the noise of the HgCdTe APD detector, the noise test system is based on Fourier transform noise spectrum analyzer, as shown in Figure 1, the background noise level of the system is about $2 \times 10^{-28} \mathrm{~A}^{2} / \mathrm{Hz}$, which can be used to characterize the noise level of devices. When a certain bias voltage is applied to the device, the current signal of the device is amplified by the preamplifier and then input to the noise spectrum analyzer of Fourier transform to process and analyze the test data(Li hao,Lin chun,et al. 2019). 


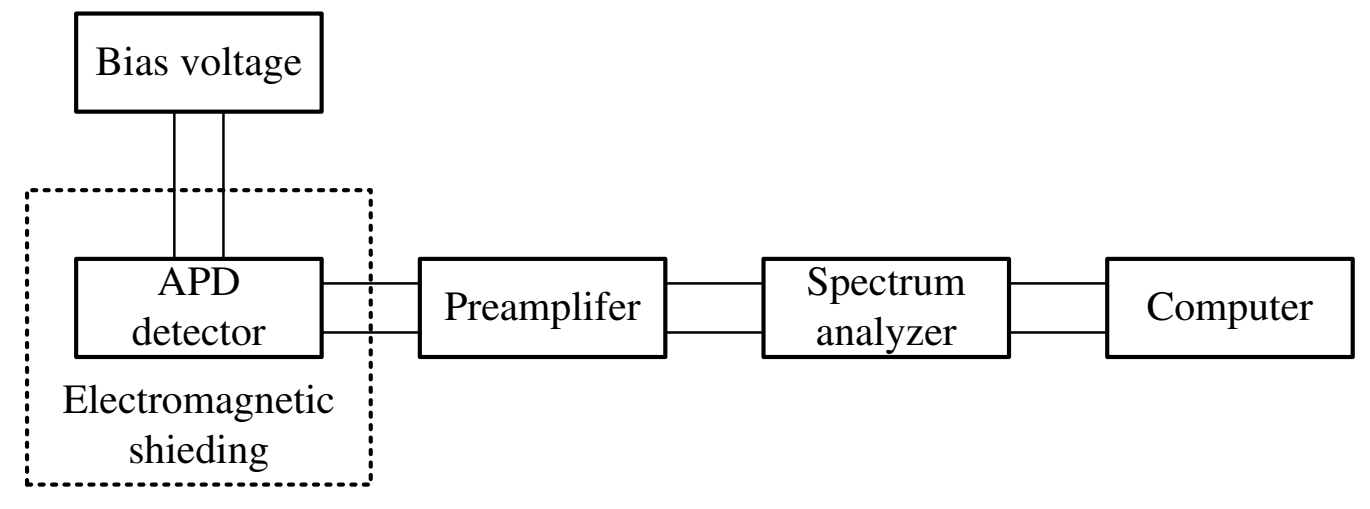

Fig. 1 Schematic diagram of noise measurement system

The low-frequency noise test results of $\mathrm{HgCdTe}$ APD unit devices are shown in Figure 2, the Cd composition of the $\mathrm{HgCdTe}$ material use in the device is 0.269 , corresponding to the cut-off wavelength of $6.4 \mu \mathrm{m}$ at $77 \mathrm{~K}$ and the P-type $\mathrm{Hg}$ vacancy concentration of $6 \times 10^{15} \mathrm{~cm}^{-3}$, it can be seen that the $1 / \mathrm{f}$ noise component of the device becomes more and more obvious as the voltage increases.

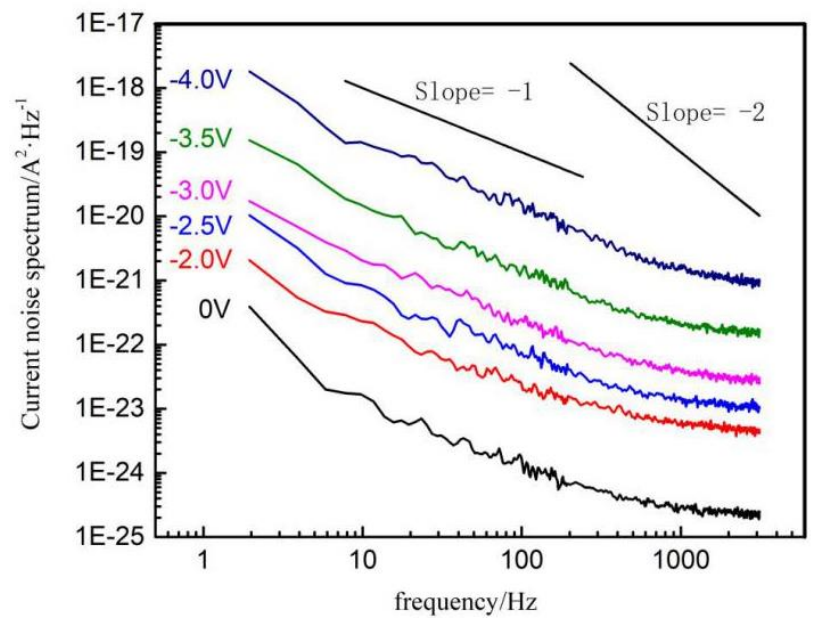

Figure 2 Noise PSD of HgCdTe APD unit device

Gain Normalized Dark Current Density (GNDCD) use to characterize the dark current changes of APD devices when avalanche multiplication occurs, as shown in Figure 3, The device state is stable below $6 \mathrm{~V}$, and GNDCD changes smoothly. Starting from $8 \mathrm{~V}$, due to the increase of tunneling current, the dark current also increases rapidly. The excess noise factor $\mathrm{F}$ also shows the same trend. It is about 1.2 under a small bias, and the calculated $\mathrm{F}$ is much higher than the normal value when the increase of the tunneling current at $8 \mathrm{~V}$. Through the noise test and analysis of the detector, it provides a basis for reducing the influence of noise on the circuit. 


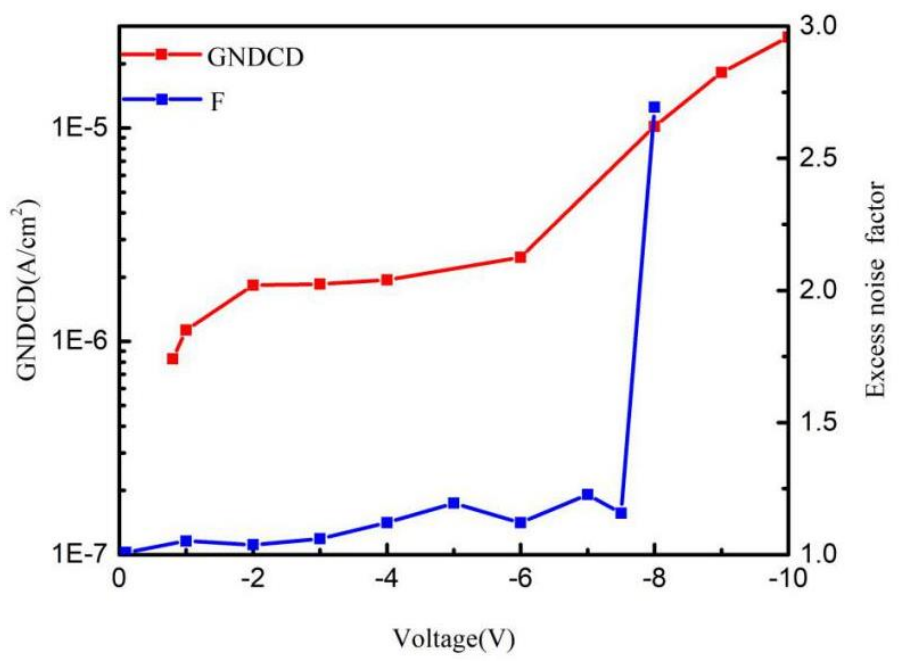

Figure 3 Excess noise factor of APD FPA and GNDCD with different bias

\section{Analysis of readout circuit structure}

The structure design of the focal plane readout circuit is shown in Figure 4. It is mainly composed of pre-amplification, sample-and-hold, time-to-digital conversion circuit (TDC), phase locked loop (PLL) and control circuit. Its working principle is that when the laser pulse is emitted, the synchronous trigger circuit TDC starts to work, when the detector detects the return laser pulse, the circuit quickly integrates so that the voltage reaches the TDC comparator threshold and flips, generating a stop signal, and the TDC carries the time The analog signal of the information is converted into a digital signal, thereby quantifying time and realizing three-dimensional ranging. Its accuracy will determine the minimum time resolution.

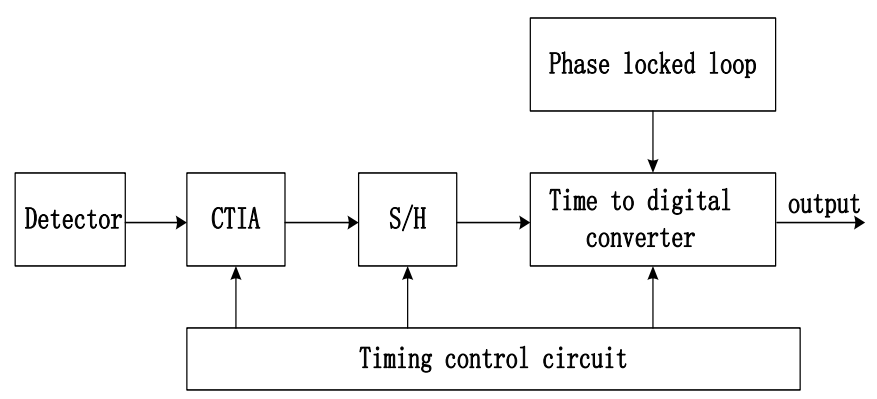

Fig 4 HgCdTe APD ROIC overall block diagram

TDC circuit adopts the vernier type structure, comparing with other structures, it has the advantages of high precision, large dynamic range and easy control. The circuit structure and timing are shown in Figure 5. which consists of a control circuit, a vernier delay line circuit (coarse and fine counting circuits), a 
comparator, a shift register readout circuit, et.al.

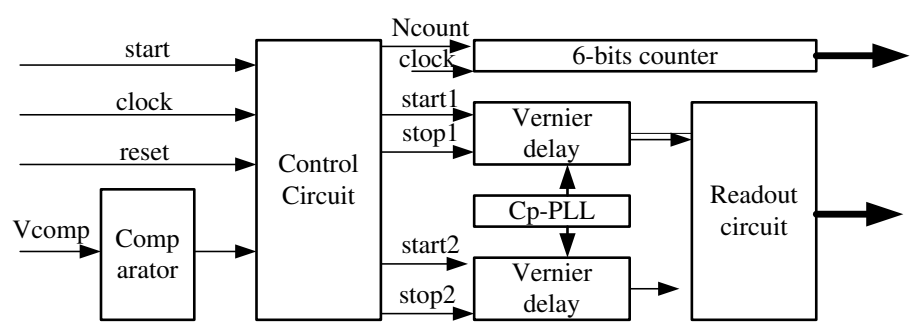

(a)

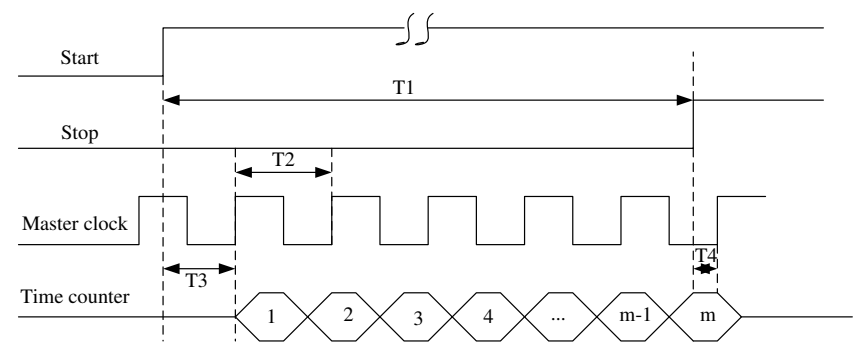

(b)

Fig.5 (a) TDC circuit functional block diagram; (b) TDC sequence chart

The sequence of the working principle for the vernier type TDC is shown in Figure 5(b). The time T1 between the start signal and the end signal consists of three parts: (1) the time interval T3 between the start signal START and the next rising edge of the counter clock, (2) the clock period T2, and (3) the time interval T4 between the end signal STOP and the next rising edge of the clock.Thus, time T1 can be written as:

$$
T 1=m * T 2+T 3-T 4
$$

Where $\mathrm{m}$ indicates the number of $\mathrm{T} 2$. The time interval accuracy determines the resolution of the time-to-digital conversion circuit.

The structure of the fine counting vernier delay circuit is shown in Figure 6 (Guo et al. 2012;Li 2015;Huang 2004). It consists of two delay chains (multiple identical delay units connected in series), one is for transmitting the START signal with a delay time of $\mathrm{t}$, and the other is for transmitting the STOP signal with a delay time of $\mathrm{t} 2(\mathrm{t} 1>\mathrm{t} 2)$. The time interval between the two delay chains is:

$$
T_{L S B}=t 1-t 2
$$

Each stage is sampled by DFF. Before the STOP signal catches up with the START signal, the output 
is 0 ; when the $\mathrm{N}$ stage just catches up or exceeds, the output is 1 and flip to stop counting. Therefore, the time can be written as $\mathrm{N} * \mathrm{~T}_{\mathrm{LSB}}$. Among them, the D flip-flop uses the true single-phase clock flip-flop TSPC structure (Takamoto 1993; Li 2012; Duan et al. 2019).

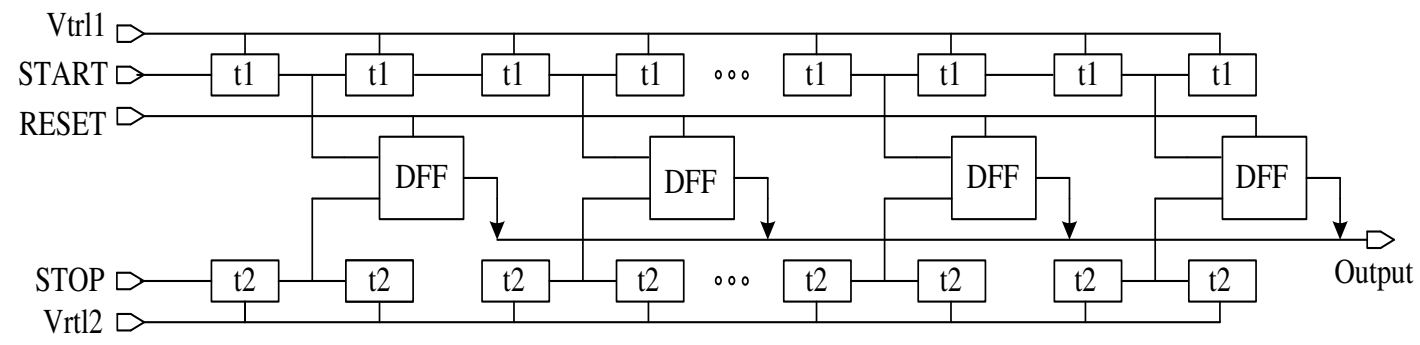

Fig.6 Vernier TDC structural diagram

The resolution of the time-to-digital conversion circuit is mainly determined by the accuracy of the comparator and the accuracy of the unit circuit in the vernier delay chain. Therefore, at $77 \mathrm{~K}$, the design of the comparator and the delay unit in the vernier delay chain are very important.

\subsection{Low temperature MOSFET model analysis}

Since the detector (HgCdTe APD) works at $77 \mathrm{~K}$, it is extremely important to study the characteristics of MOSFET devices at low temperatures. As the temperature decreases, the semiconductor ionization ability becomes weaker, and the hole or electron concentration decreases, which affects the surface potential and causes the MOSFET parameters to change: Threshold voltage increases and source-drain capacitance decreases, etc. This affects the operating characteristics of the readout circuit at low temperatures. Therefore, the establishment of a low-temperature circuit simulation model can better characterize the circuit performance.

By extracting device parameters, test MOSFET devices at $300 \mathrm{~K}$ and $77 \mathrm{~K}$, the results are shown in table 1. The main parameters of NMOS and PMOS have changed significantly at low temperatures. For example, the threshold voltage $\mathrm{V}_{\text {th } 0}$ becomes larger by $0.2 \mathrm{~V}$ at low temperature; the mobility $\mu_{0}$ becomes about three times and the unit source-drain parasitic resistance $\mathrm{R}_{\mathrm{dsw}}$ becomes about 1.5 times; the saturation speed $\mathrm{v}_{\text {sat }}$ increases about 3 times for PMOS.

Table 1 Extraction results of the MOSFET parameters 


\begin{tabular}{|c|c|c|c|c|c|}
\hline \multirow{2}{*}{ Model parameters } & \multirow{2}{*}{ Unit } & \multicolumn{2}{|c|}{ NMOS } & \multicolumn{2}{|c|}{ PMOS } \\
\hline & & $77 \mathrm{~K}$ & $300 \mathrm{~K}$ & $77 \mathrm{~K}$ & $300 \mathrm{~K}$ \\
\hline $\mathrm{V}_{\text {th0 }}$ & V & 0.958 & 0.719 & -1.121 & -0.724 \\
\hline$\mu_{0}$ & $\mathrm{~cm}^{2} / \mathrm{Vs}$ & 1370 & 434 & 564 & 172 \\
\hline $\mathrm{v}_{\mathrm{sat}}$ & $\mathrm{m} / \mathrm{s}$ & $9.59 \mathrm{E} 4$ & 7.47E4 & $9.61 \mathrm{E} 4$ & $3.81 \mathrm{E} 4$ \\
\hline $\mathrm{R}_{\mathrm{dsw}}$ & $\Omega-\mu \mathrm{m}$ & 1663.2 & 1373.16 & 3841.31 & 2530.47 \\
\hline
\end{tabular}

Simultaneously analyze the on-resistance and junction temperature characteristics of the MOSFET, The result is shown in Figure 7. Under the saturated conduction condition of the MOSFET, $R_{d s}$ tends to increase with increasing temperature, realizing MOSFET switching speed faster.

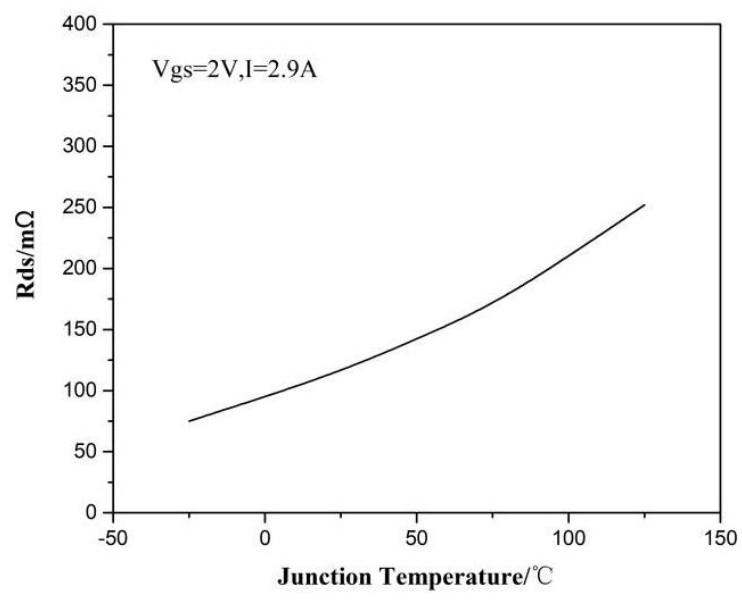

Fig.7 Relationship between MOS tube on-resistance and junction temperature

Figure8 shows the I-V curve measured at $300 \mathrm{~K}$ and $77 \mathrm{~K}$ for NMOS devices with aspect ratio $\mathrm{W} / \mathrm{L}=20 \mu \mathrm{m} / 0.55 \mu \mathrm{m}$. Under the same gate-source voltage Vgs, the operating current in the saturation region of the NMOS device at $77 \mathrm{~K}$ is significantly higher than that at room temperature, It provides a basis for modeling at low temperature. 


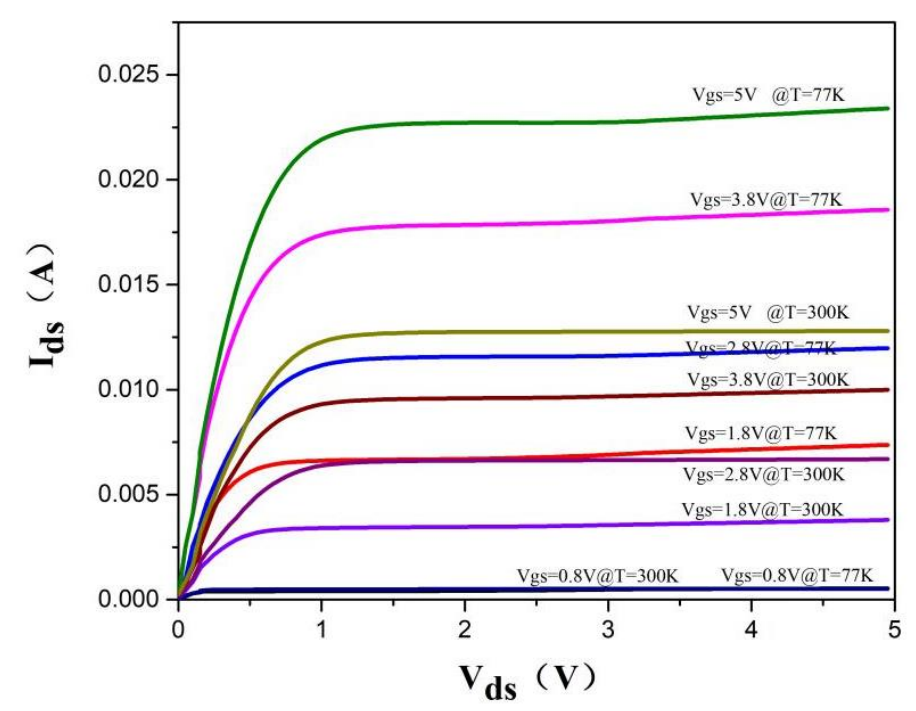

Fig.8 I-V curves of NMOS at $77 \mathrm{~K}$ and $300 \mathrm{~K}$. The size of the NMOS is $\mathrm{W} / \mathrm{L}=20 \mu \mathrm{m} / 0.55 \mu \mathrm{m}$

\subsection{Delay unit circuit design}

Since the propagation of the delay unit composed of the traditional two-stage inverter is easily affected by PVT, the relationship between them can be written as:

$$
\frac{\delta T_{D}}{\delta P_{d e v}}=-\frac{C_{L} V_{D D}}{2 \frac{W}{L_{e f f}} P_{d e v}^{2}}
$$

where, $\mathrm{W}$ and $\mathrm{L}_{\text {eff }}$ are the gate width and equivalent channel length of the transistor respectively, and $\mathrm{P}_{\mathrm{dev}}$ is the degree of influence the device parameters on the circuit. Therefore, measures to reduce the propagation delay of the inverter: reduce $C_{L}$, increase W/L of the MOS tube, that is, reduce $R_{\text {eqn }}$ and $R_{\text {eqp }}$ and increase VDD. It is easy to get that in order to make the circuit more stable, the value of $\mathrm{W}$ can be increased appropriately when the channel length is unchanged. Therefore, in our designing, the aspect ratio (i.e., W/L) of the NMOS and PMOS tube are set to 2:1 and 4:1, respectively.

The delay unit circuit adopts a voltage-controlled structure, as shown in Figure 9. By adjusting the voltage Vtrl of the bias tube, different delays can be obtained. the two NMOS tubes are connected to the power supply voltage to ensure that it can be used in at all process angles. The desired delay time within the corresponding range is obtained by adjusting the bias voltage Vtrl. At the same time, a symmetrical structure is adopted in the delay unit to reduce the influence caused by the parasitic effect of the layout. 


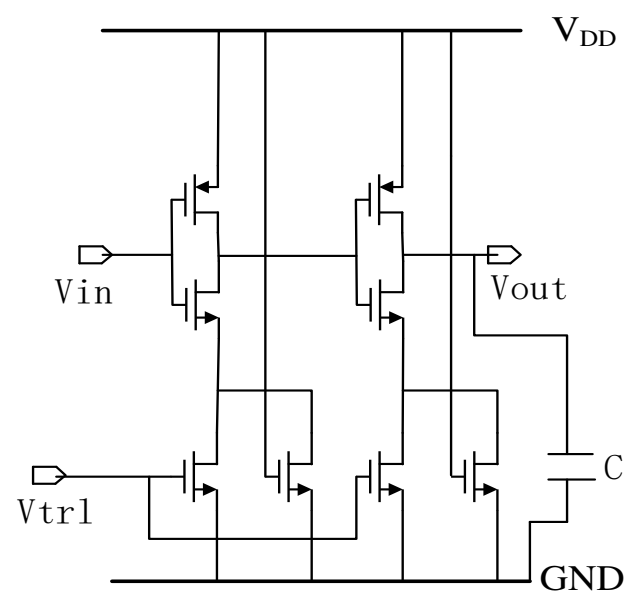

Fig.9 delay cell circuit

During the simulation, the bias voltage is scanned from 0 to $5 \mathrm{~V}$, For comparison, the simulation temperature is set to room temperature and $77 \mathrm{~K}$, respectively,then we can get the delay time values under the two different temperatures as a function of the bias voltage, the results are shown in Figure 10.

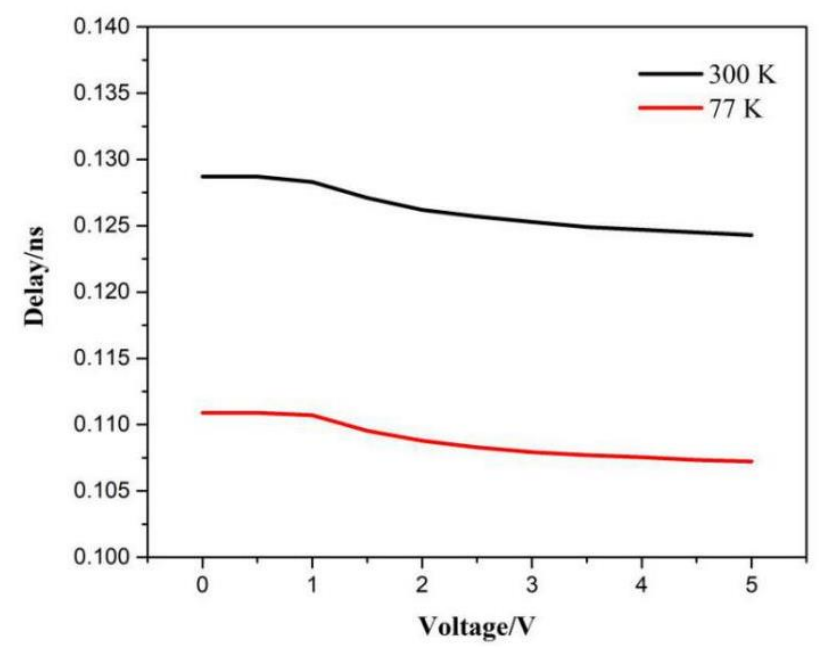

Fig.10 Delay time at different temperatures and different bias voltages

It can be seen from the Figure 10 that the delay unit can easily achieve smaller time resolution at low temperatures .

\subsection{Comparator design}

As shown in Figure 11, the comparator adopts high-speed comparator structure, which can better combine the advantages of an open-loop comparator and a regenerative comparator. It hence has the advantages of high precision and low offset. The preamplifier amplifies the input differential signal and 
improves the resolution of the comparator. Its own isolation effect makes the comparator have smaller kickback noise and input offset voltage. The latch (hysteresis) structure is the core, which can effectively increase the speed of the comparator and determine the resolution accuracy. The purpose of the self-biased differential amplifier is to drive the load. The simulation show that the self-biasing working state is between 1 to $3 \mathrm{~V}$. so , in our design, we add an NMOS tube is added to the latch part of the design to raise the minimum voltage of the latch, and make the self-biasing circuit works in a normal state.

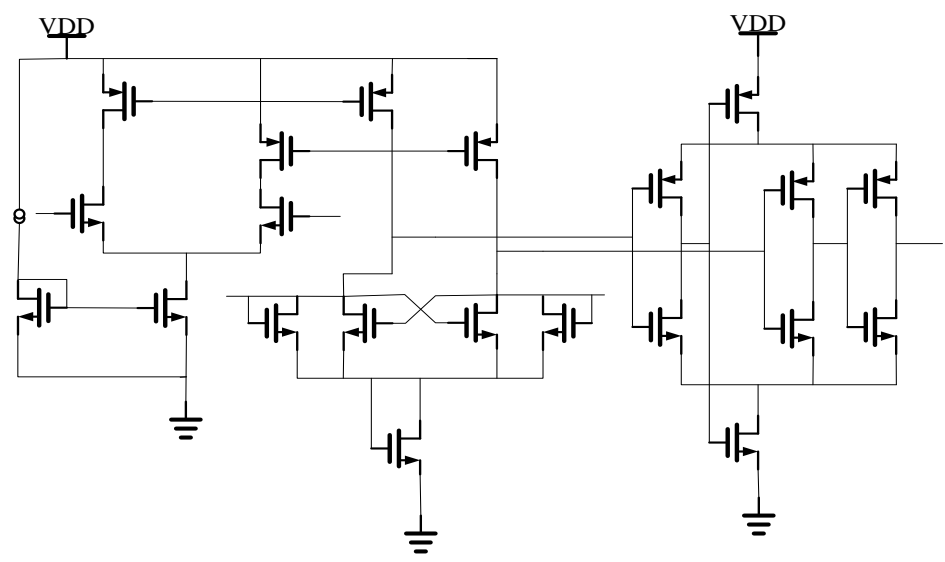

Fig.11 Comparator schematic diagram

As we all know that the accuracy of the comparator is directly affected by the input offset voltage.thus in our design, the offset voltage is mainly composed of two parts, i.e.,the input voltage of the pre-amplifier and the latch, namely:

$$
\sigma=\sqrt[2]{\sigma_{\text {vospreamp }}^{2}+\frac{1}{A_{V}^{2}} \sigma_{\text {voslatch }}^{2}}
$$

Here, $A_{V}$ is the gain of the pre-amplifier, so the offset voltage of the pre-amplifier greatly affects the offset voltage of the entire comparator.

The transistors which in the pre-amplifier contributes to the offset are mainly the input tubes M1 and M2 and the load tubes M5 and M6. namely:

$$
\begin{gathered}
\sigma_{\text {offset }}^{2}=\sigma_{1.2}^{2}+\sigma_{5.6}^{2} \\
\sigma_{1.2}^{2} \approx \frac{g_{m 1.2}}{g_{m 7.8}}\left[\frac{A_{V T P}^{2}}{W L}+\left(\frac{V_{D S 1.2}^{2}}{4} * \frac{A_{\beta P}^{2}}{W L}\right)\right]
\end{gathered}
$$




$$
\sigma_{5.6}^{2} \approx \frac{\mathrm{A}_{\mathrm{VTN}}^{2}}{\mathrm{WL}}+\left(\frac{\mathrm{V}_{\mathrm{DS} 5.6}^{2}}{4} * \frac{\mathrm{A}_{\beta \mathrm{N}}^{2}}{\mathrm{WL}}\right)
$$

Where $A_{V T N}$ and $A_{V T P}$ are the threshold voltage offset factor of the process model, $A_{\beta N}$ and $A_{\beta P}$ are the offset of the process factor. It can be seen from the formula that with increasing the tube size the effect of the imbalance can be reduced.

Usually increasing the gain of the amplifier can also reduce the offset noise. Since the gain bandwidth product is a constant, and in order to ensure that the bandwidth of the comparator is above $100 \mathrm{MHz}$, the gain of the pre-amplifier stage in the design cannot be too large, so the gain is designed to be $6.02 \mathrm{~dB}$ in the circuit.

In the simulation, the comparator reference voltage is $2 \mathrm{~V}$, and a ramp voltage is applied to the other end. The ramp voltage is from $1 \mathrm{~V}$ to $3 \mathrm{~V}$ has a time interval of $1.06 \mathrm{~ms}$. The most used methods in the simulation are Monte Carlo and corner simulations. Figure 12 shows the worst case in the corner simulation when the comparator is selected under CMOS technology at $77 \mathrm{~K}$, and the different states of tt, ff, fs, sf, ss, etc..It can be seen that the equivalent input offset voltage is $0.45 \mathrm{mV}$. The maximum offset voltage at less than $1 \mathrm{LSB}$ is $0.244 \mathrm{mV}$.Table 2 shows the parameter simulation results of the hybrid comparator.

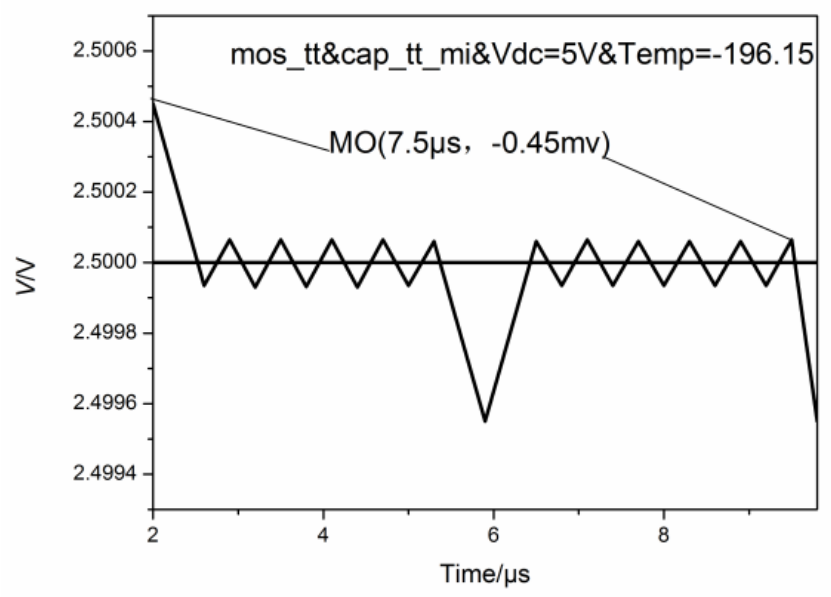

Fig.12 Simulation results of input offset voltage of comparator

Tab.2 comparator simulation results

\begin{tabular}{cc}
\hline Gain & $77.99 \mathrm{~dB}$ \\
\hline Delay Time & $10.01 \mathrm{~ns}$ \\
\hline
\end{tabular}




\begin{tabular}{lc}
\hline Resolution & 12 bits \\
Noise & $7.621 \mathrm{v} / \mathrm{sqrt}(\mathrm{Hz}) @ 1 \mathrm{MHz}$ \\
\hline
\end{tabular}

\subsection{Unit circuit simulation results}

The output structure of the TDC vernier delay chain is 0 and 1 , and the resolution depends on the time interval which is less than one clock cycle between the start/stop signal and its adjacent main clock rising edge. Under the master CLK of $120 \mathrm{MHz}$, the simulation parameters are set, and the start signal start changes to high level at $1.15 \mu \mathrm{s}$. By comparing with the rising edge of the next clock cycle, the time interval is $6.6 \mathrm{~ns}$, and the applied bias voltages of the two delay chains are $1 \mathrm{~V}$ and $4 \mathrm{~V}$ respectively. Based on the previous analysis of the resolution of the delay unit and the comparator, the theoretically calculated accuracy that can be achieved is:

$$
T_{R S}=\frac{R}{U}+D_{t}=\frac{1.06 u s * 0.224 * 10^{-3} / 2}{128}+40 p s=132.75 p s
$$

Where, $\mathrm{R}$ is the resolution accuracy of the comparator, $\mathrm{U}$ is the total number of the delay units, and $\mathrm{D}_{\mathrm{t}}$ is the accuracy of the delay units.

At $77 \mathrm{~K}$, we use the CSMC $0.5 \mathrm{~m} 2 \mathrm{P} 3 \mathrm{M}$ process to do simulation, the simulation results are shown in Table 3. It shows that the value near the flip point, and the 50th flip is achieved in the counter. Which means the accuracy of $132 \mathrm{ps}$ can be achieved in our simulation. Comparing the theoretical value with the simulated value, the theoretical value of $132.75 \mathrm{ps}$ is between the simulated value of $132 \mathrm{ps}$ and 6.6ns $/ 49=134.69$ ps. Thus, between 49 and 50, the simulation results are in good agreement with the theoretical calculation results.

Tab.3 Vernier TDC simulated output

\begin{tabular}{cc}
\hline COUNTER & LEVEL \\
\hline 1 & 0 \\
2 & 0 \\
$\ldots$ & $\ldots$ \\
\hline
\end{tabular}




\section{Experimental results}

After the circuit is taped out, the test chip is packaged in QFP64, the test chip is placed on a custom-designed printed circuit board (PCB), The PCB is assembled in a laboratory Dewar which can operate with liquid nitrogen(N2), As shown in Figure 13.

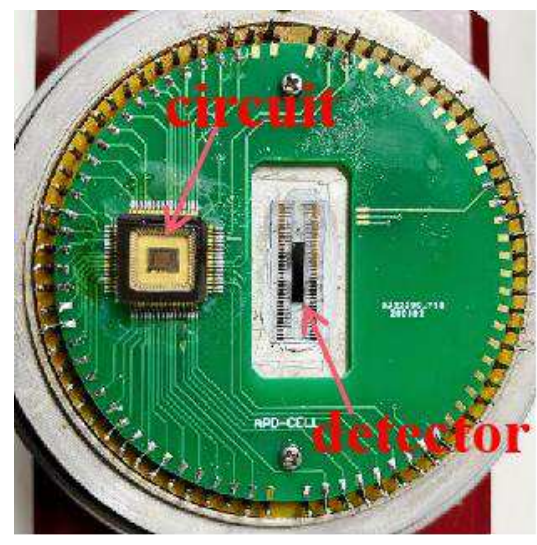

Fig.13 Tested chip in the Dewar

The block diagram of the test system is shown in Figure 14. It is composed of a high-speed DIO system on a computer, a NI6552 digital timing generator, an Agilent voltage source, a NI6552 digital acquisition card, a chip under test and an oscilloscope. NI6552 has 20 channels, which can realize digital timing transmission and acquisition. The voltage source provides bias for the chip, and the high-speed DIO system controls the NI6552 for timing transmission and acquisition. The test is divided into two parts: room temperature test and $77 \mathrm{~K}$ test. 


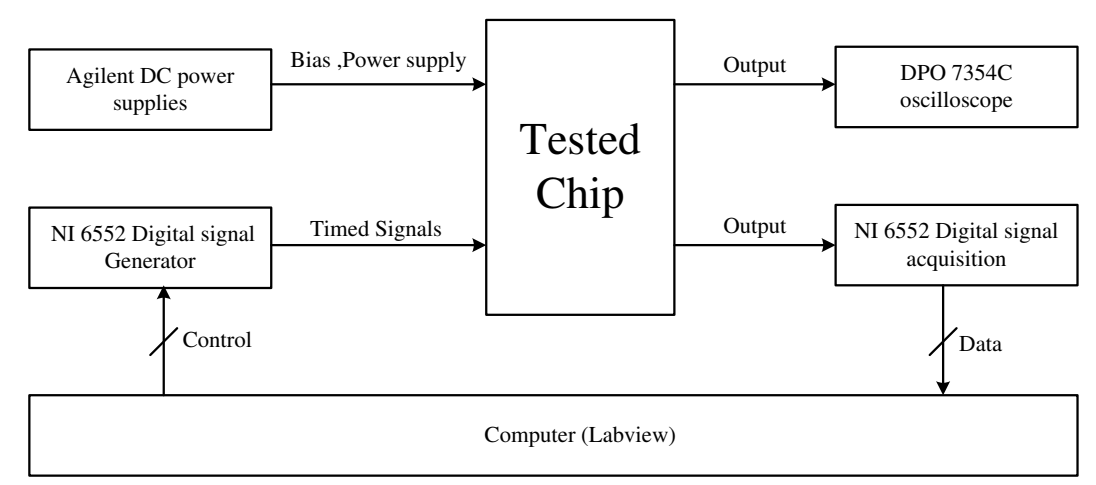

Fig.14 Schematic block diagram of TDC circuit Testing system

Experiment with TDC at room temperature and at $77 \mathrm{~K}$ respectively, the test sequence is shown in Figure 15. Master_CLK is the main clock signal of the circuit, RST is the reset signal to reset the circuit, Start signal and Stop signal are the start and end signals of counting respectively,the difference between them is $850 \mathrm{~ns}$. Shift CP is the clock signal of the shift register, and Shift pulse is the shift pulse signal.

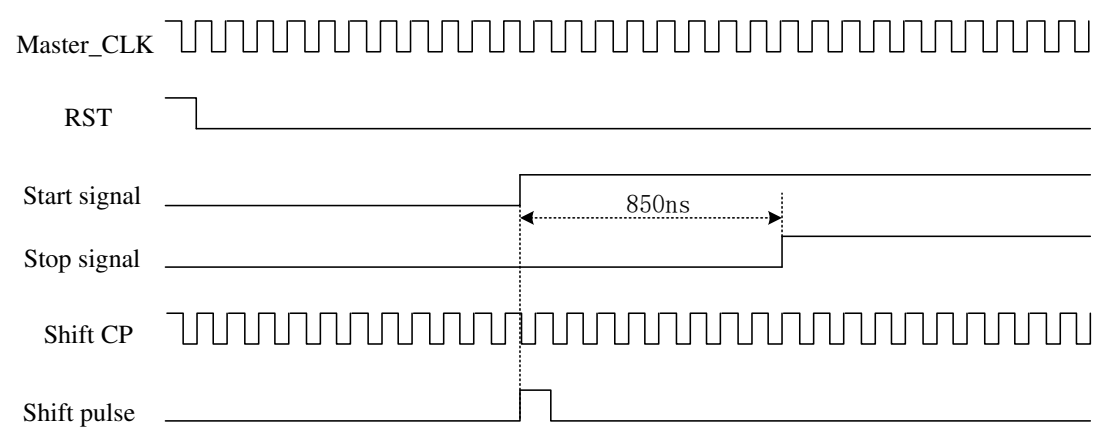

Fig.15 Test sequence diagram

The coarse count is composed of a 6-bit shift register, and the results are consistent with repeated times at room temperature and $77 \mathrm{~K}$, as shown in Figure 16.

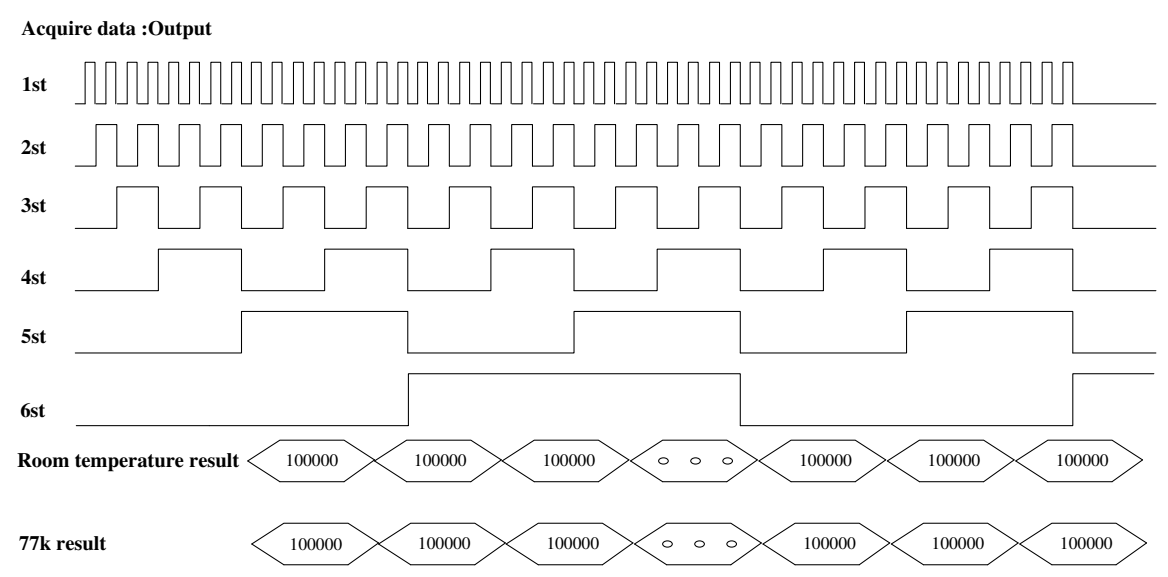


Fig. 16 coarse counting test results at room temperature and low temperature

The results of the precision counting test are shown in Figure 17. We find that, within $6.6 \mathrm{~ns}$, it takes an average of 22.187 cycles to catch up at room temperature, the resolution converted to TDC is $297.472 \mathrm{ps,}$ and its RMS value is $47.235 \mathrm{ps}$; however, it takes 27.933 cycles to catch up at low temperature, the conversion time is able to achieve an resolution of $236.280 \mathrm{ps}$, and its RMS value is $26.709 \mathrm{ps}$.

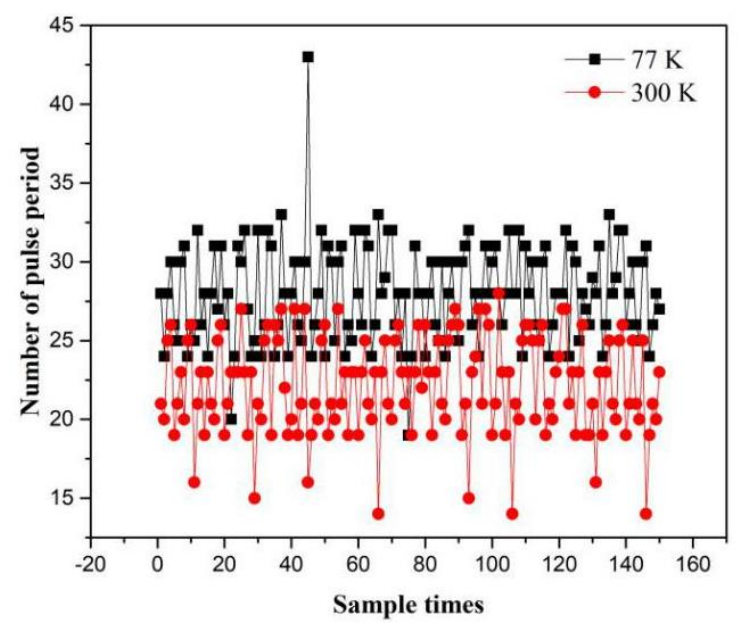

Fig.17 Fine counting value at room temperature and low temperature test

It can also be seen from the Figure 12 that a deviation of the catch-up period appears in the repeated experiment, it leads to the non-linearity of the integral. For the high-frequency digital part, the parasitic effect has a great influence on the circuit, it can cause an increasing of the delay time of the delay unit; In the actual measurement, the error caused by the jitters of the internal clock multiplication CP-PLL directly affects the total time value which less than one cycle and the offset error of the comparator also affects the accuracy.

With using origin pro to fitting our test data, we further obtain the degrees of the differential and integral non-linearity, see Figure 18 below. The values of the degrees are -0.54 0.71 LSB and -1.32 1.21 LSB, respectively. Thus, finally we can get that $|\mathrm{DNL}|$ that $|\mathrm{INL}|$ of TDC is less than 1LSB and 2LSB, respectively. 


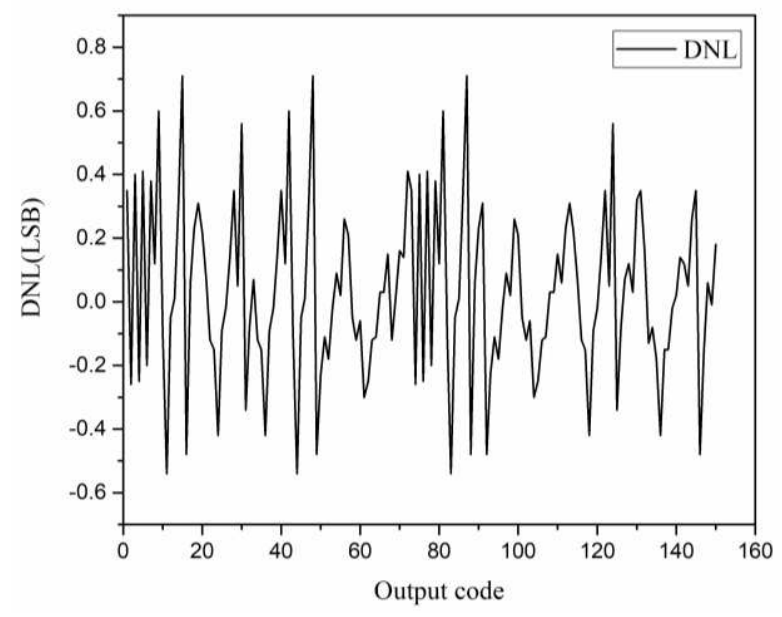

(a)

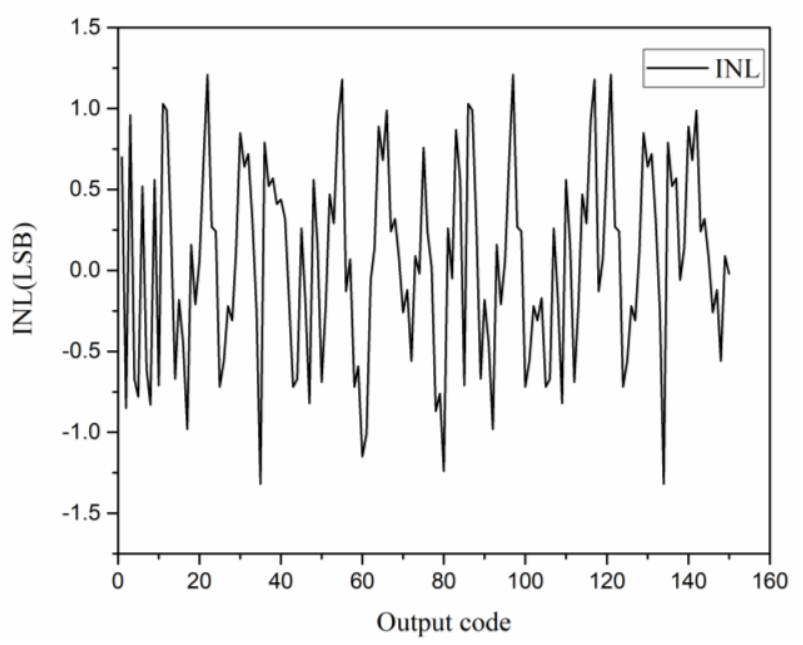

(b)

Fig.18 (a) Differential non-linearity;(b)Integral non-linearity

At $77 \mathrm{~K}$ and $300 \mathrm{~K}$, The simulation test summary of the whole circuit parameters is shown in Table 4 below, and compare with Raytheon.

Tab.4 Compare this work and Raytheon at $77 \mathrm{~K}$ and $300 \mathrm{~K}$

\begin{tabular}{cccc}
\hline Performance parameter & \multicolumn{2}{c}{ This work } & Raytheon(William McKeag et al) \\
\hline Technology & CSMC CMOS 0.5um 2P3M & CSMC CMOS 0.5um 2P3M & TSMC CMOS 0.18um \\
Temperature & $77 \mathrm{~K}$ & $300 \mathrm{~K}$ & $77 \mathrm{~K}$ \\
Resolution & $236.28 \mathrm{ps}$ & $297.47 \mathrm{ps}$ & $166.67 \mathrm{ps}$ \\
\hline
\end{tabular}




\begin{tabular}{lccc}
\hline Dynamic Range & 12 Bits & 12 Bits & 12 Bits \\
Supply Voltage & $5 \mathrm{v}$ & $5 \mathrm{v}$ & $1.8 \mathrm{~V}$ \\
\hline
\end{tabular}

\section{Conclusion}

In this work, we analyzed the performance of the time-to-digital conversion circuit of the mercury cadmium telluride APD detector at $77 \mathrm{~K}$. The parameters that affect the resolution are mainly determined by the accuracy of the delay unit of the vernier delay chain and the high-speed comparator. The test results of our circuit simulation indicates that changing the difference of the delay chain via controlling its bias voltage as well as using a high-speed comparator with a latch structure can improve the accuracy of the circuit. At $77 \mathrm{~K}$, the resolution of 236.28ps is obtained, for TDC, and the degree of the integral and differential non-linearity are $-0.54 \sim 0.71$ LSB and $-1.32 \sim 1.21$ LSB, respectively( $|\mathrm{DNL}|<1 \mathrm{LSB}$, $|\mathrm{INL}|<2 \mathrm{LSB})$.

\section{References:}

A Roga lsk,i J Antoszew sk,i L Faraone. Third-generation infrared photodetector arrays [J]. Journal of Applied Physics,2009, 105( 9) :091101- 1- 091101- 44.

LU Wu,YE Zhen-hua.Development Trends of Infrared Photodetectors Abroad[J].Laser and Infrared, 2011, 41(4):365-371.

Properties of the HgCdTe Linear-Mode e-APD J. Beck, M. Kinch, R. Scritchfield, M. Woodall, M. Ohlson, L. Wood, P. Mitra, J. Robinson DRS RSTA Inc.

Phillip E.Allen. CMOS Analog Circuit Design [M].BEIJING: Publishing House of Electronics Industry, 2012:393-396.

LUO Min.Research summary of time to digital converter[J]. Microelectronics,2014,44(3):372-375.

FENG Zhi-feng.High-accuracy TDC for laser range finder[J]. Optics and Precision Engineering, 2010,18(12):2665-2671.

G.H.LI and H.P.Chou.A high resolution time-to-digital converter using two-level vernier delay line 
technique[J].IEEE, 2007:276-280.

Rothman, J., de Borniol, E., Gravrand, O., Kern, P., Feautrier, P., et al. MCT APD focal plane arrays for astronomy at CEA-LETI[J].SPIE Astronomical Telescopes, 2016, 99150B:1-12.

Jack, Michael, et al. HgCdTe APD-based linear-mode photon counting components and ladar receivers[J]. Proc. SPIE 2011, Advanced Photon Counting Techniques V, 8300: 80330M-1:80330M-18; doi: $10.1117 / 12.888134$

Li hao,Lin chun,et al. HgCdTe avalanche photodiode FPA[J]. JOURNAL OF INFRARED AND MILLIMETER WAVES,2019,38(5):587-590. doi:10.11972/j.issn.1001-9014.2019.05.007.

Lixia Zheng,Huan Hu,Ziqing Weng.Compact Active Quenching Circuit for Single-Photon Avalanche Diodes Arrays.Journal of circuits,Systems, and Computers.

Jian Guo and Sameer Sonkusale. 22-bit 110ps Time-Interpolated Time-to-Digital Converter [J].IEEE, 2012,: 3166-3169.

LI Qian-feng.Design and Implementation of time-to-digital converter in 0.18um CMOS technology[D], Nanjing:Southeast University,2015.

HUANG Zhen.Research on Receiving Circuit and Timing Method of Pulse Laser Ranging[D], Hangzhou: Zhejiang University, 2004.

Takamoto WATANABE.A CMOS Time-to-Digital Converter LSI with Half-Nanosecond Resolution Using a Ring Gate Delay Line [J]. IEICE TRANS.ELECTRON, 1993,E76-C(12):1774-1779.

LI Gen.Design of TDC based on delay locked loop[D], Harbin: Harbin Institute of Technology, 2012.

Jiangkun Duan,Maliang Liu.A 15ps resolution time-to-digital converter with on-chip PLL counting for LiDAR multi-object sensors [N]. Microelectronics Journal,2019-09-12(92).

Deng Xuguang. Low-temperature MOSFET model and parameter extraction based on BSIM3[J]. Infrared and Laser Engineering,2013,43(09):1051-1054. 
William McKeag, Tricia Veeder, Jinxue Wang, and Michael Jack. New Developments in HgCdTe APDs and LADAR Receivers[J].Infrared Technology and Applications,2011,8012(30):1-13. doi: 10.1117/12.888099. 
Figures

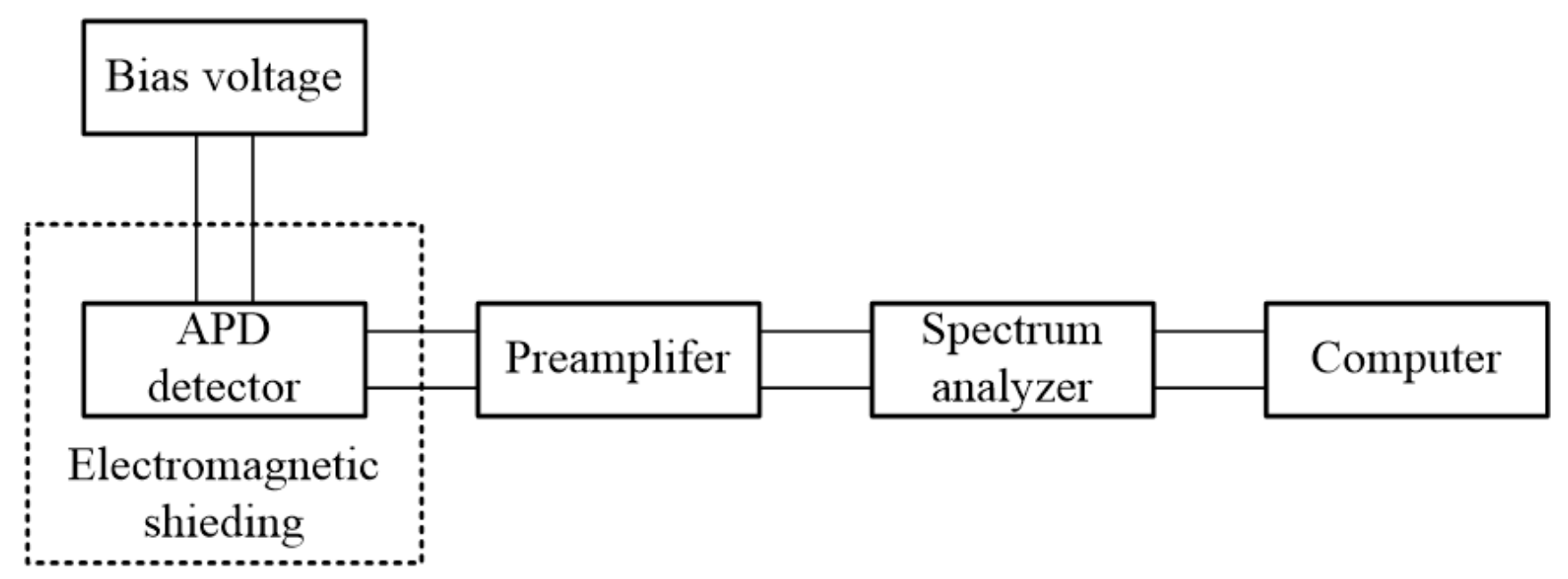

Figure 1

Schematic diagram of noise measurement system

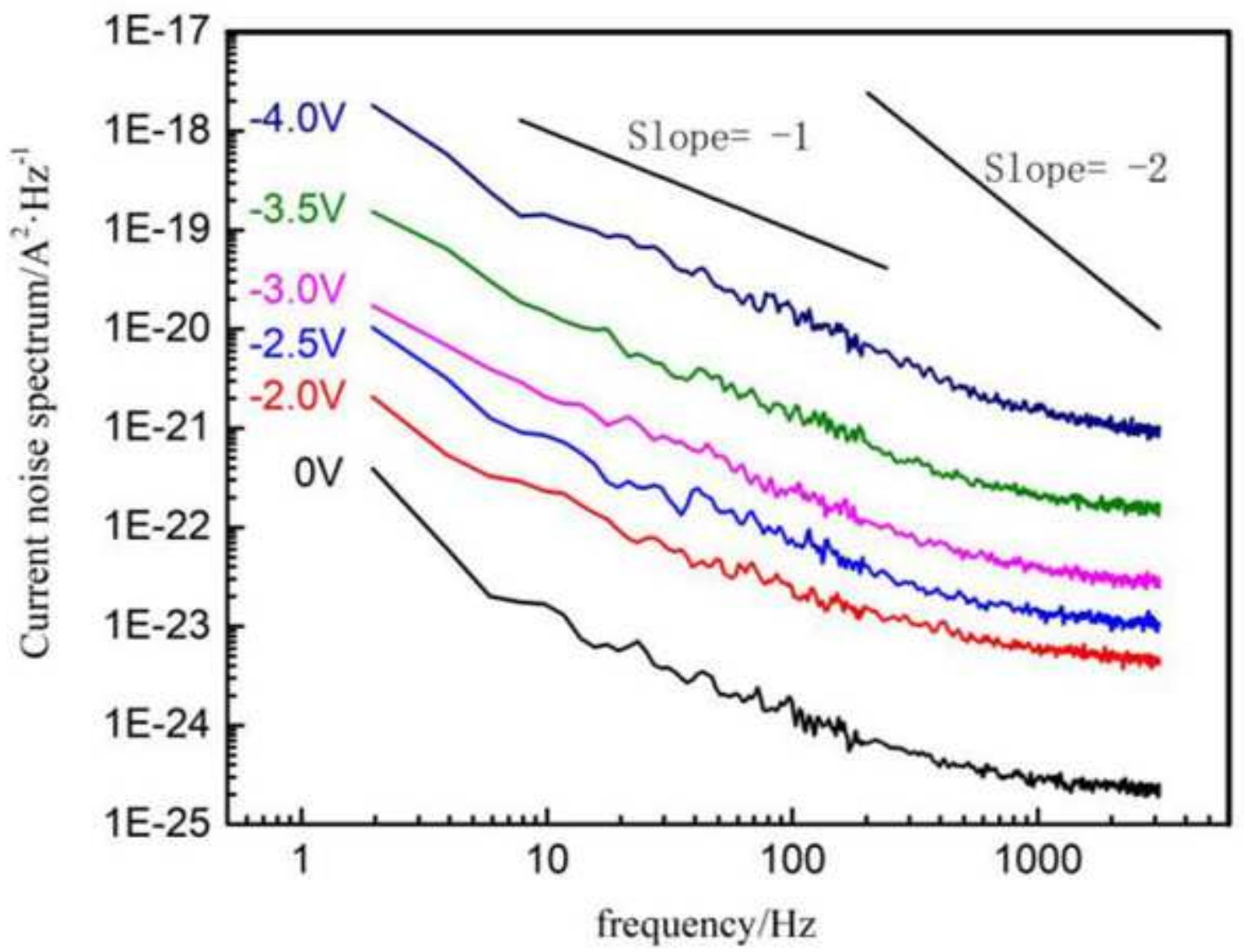


Figure 2

Noise PSD of HgCdTe APD unit device

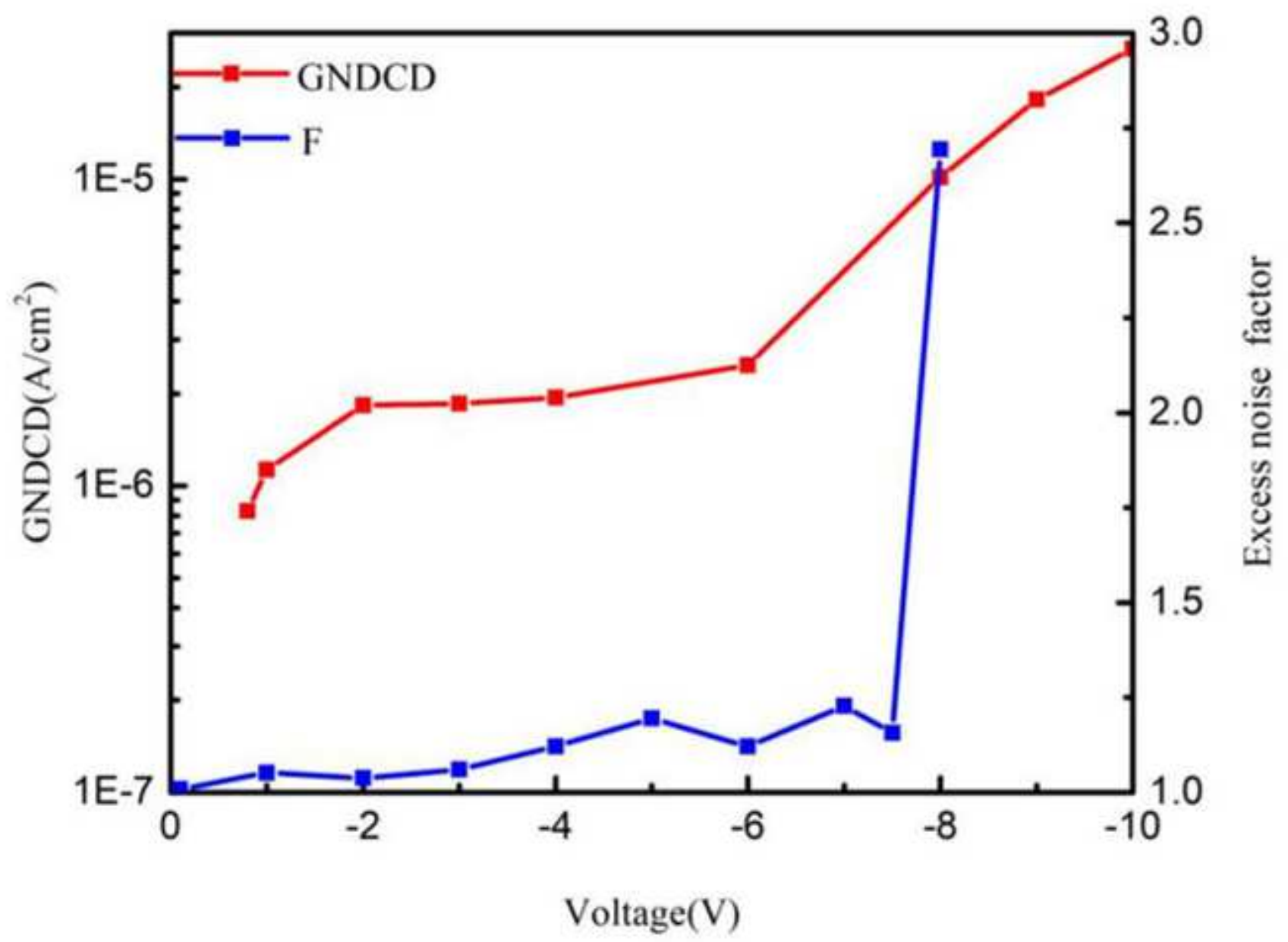

Figure 3

Excess noise factor of APD FPA and GNDCD with different bias 


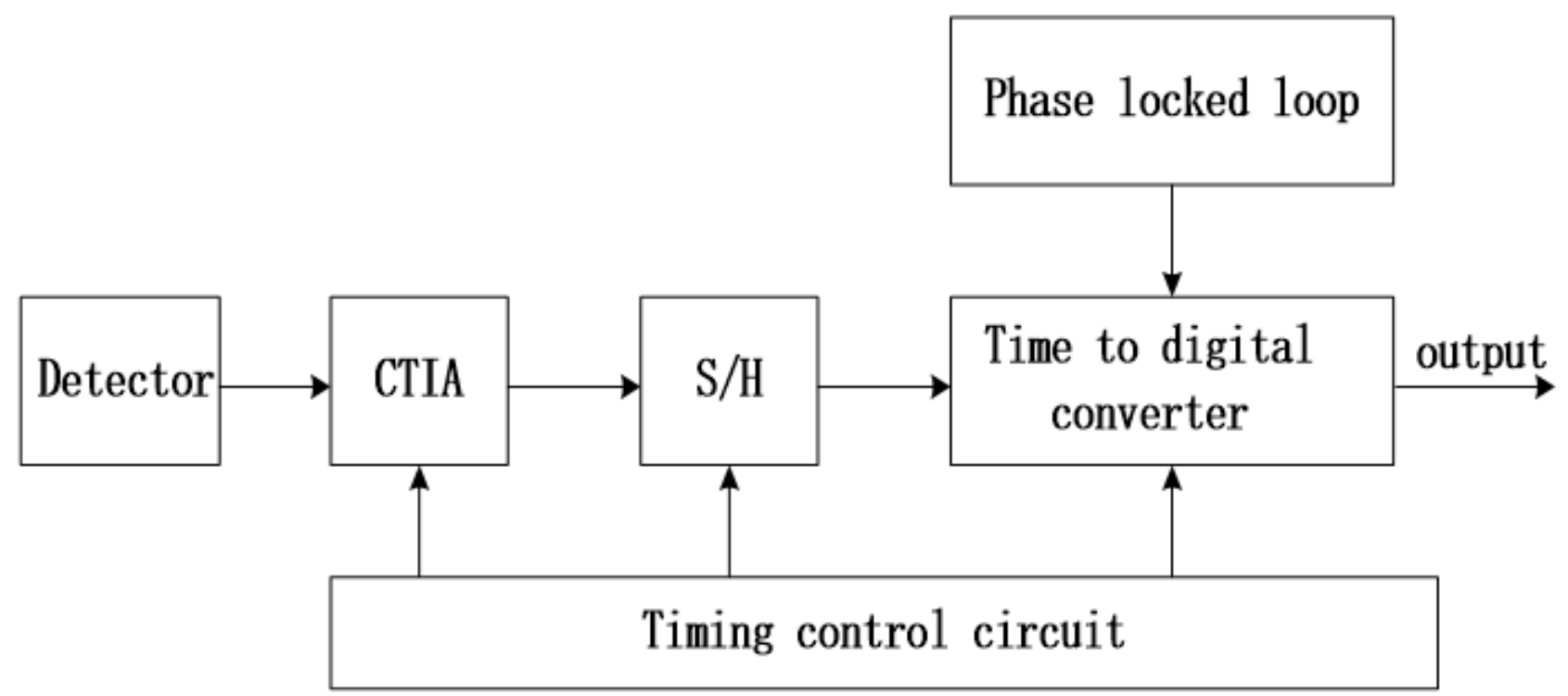

Figure 4

HgCdTe APD ROIC overall block diagram

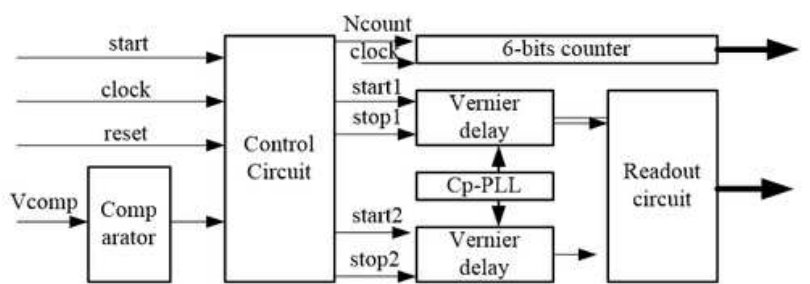

(a)

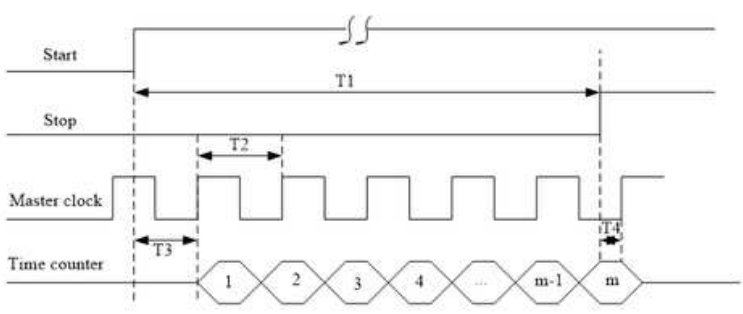

(b)

Figure 5

(a) TDC circuit functional block diagram; (b) TDC sequence chart

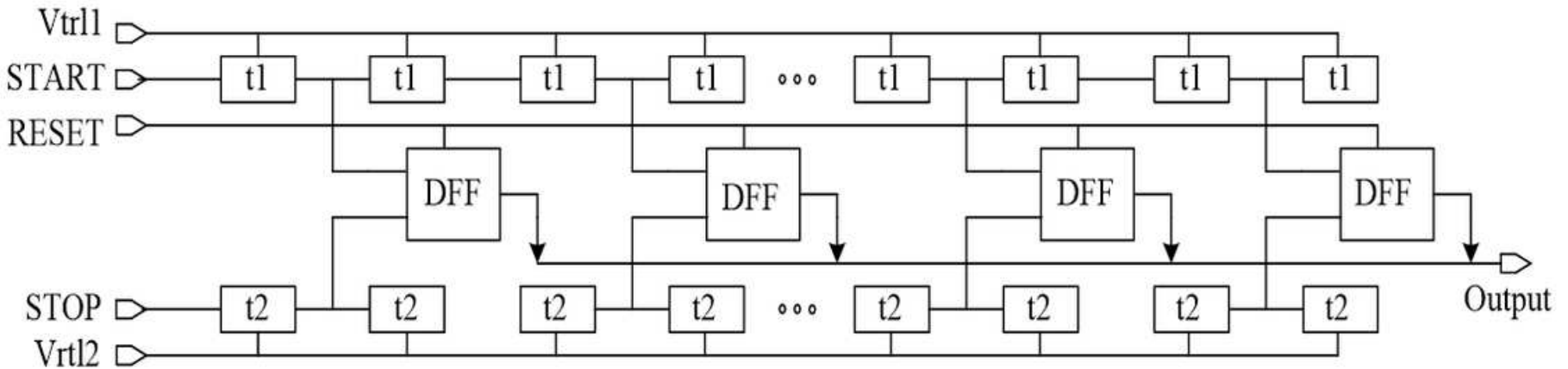


Figure 6

Vernier TDC structural diagram

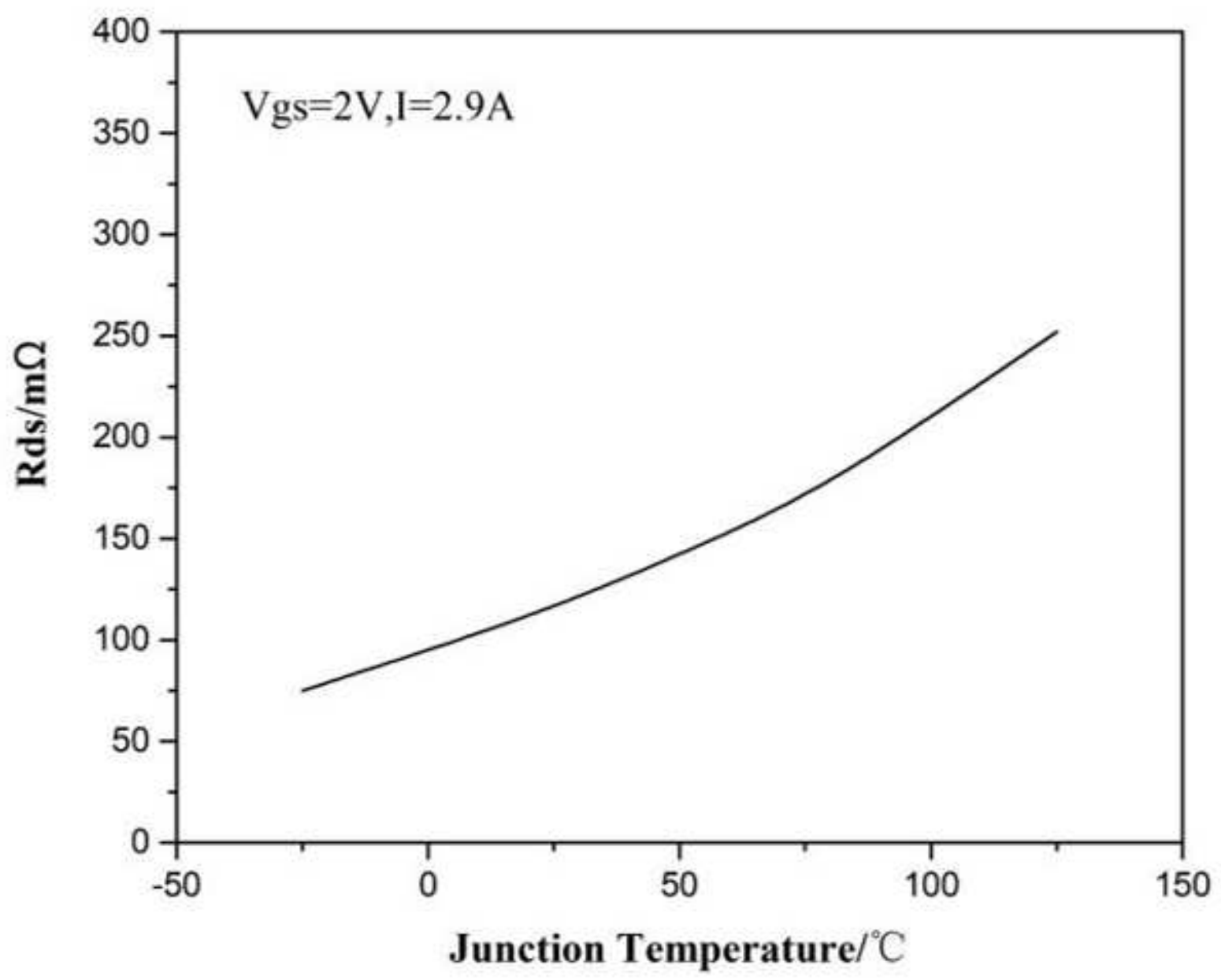

Figure 7

Relationship between MOS tube on-resistance and junction temperature 


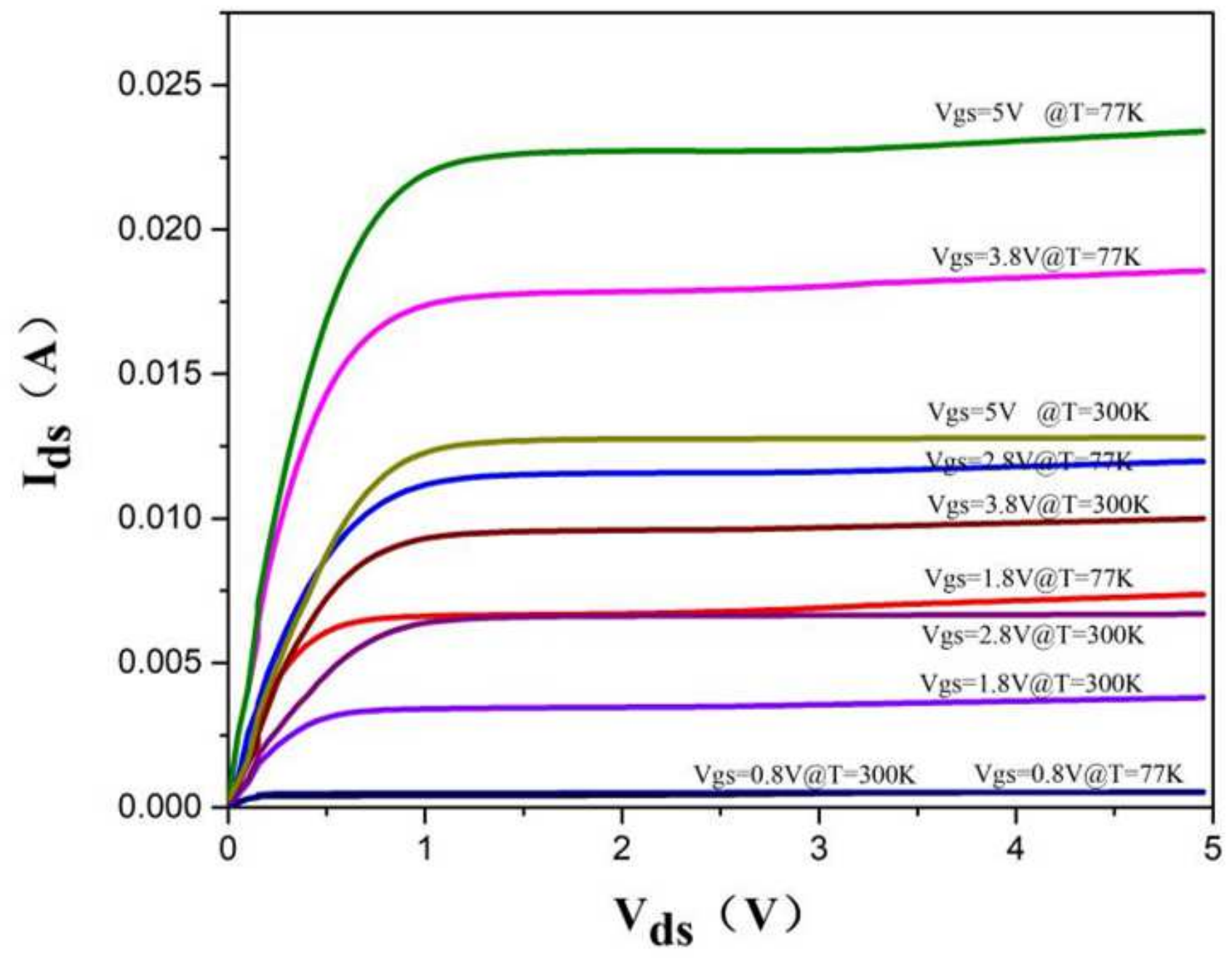

Figure 8

$\mathrm{I}-\mathrm{V}$ curves of NMOS at $77 \mathrm{~K}$ and $300 \mathrm{~K}$. The size of the NMOS is $\mathrm{W} / \mathrm{L}=20 \mu \mathrm{m} / 0.55 \mu \mathrm{m}$ 


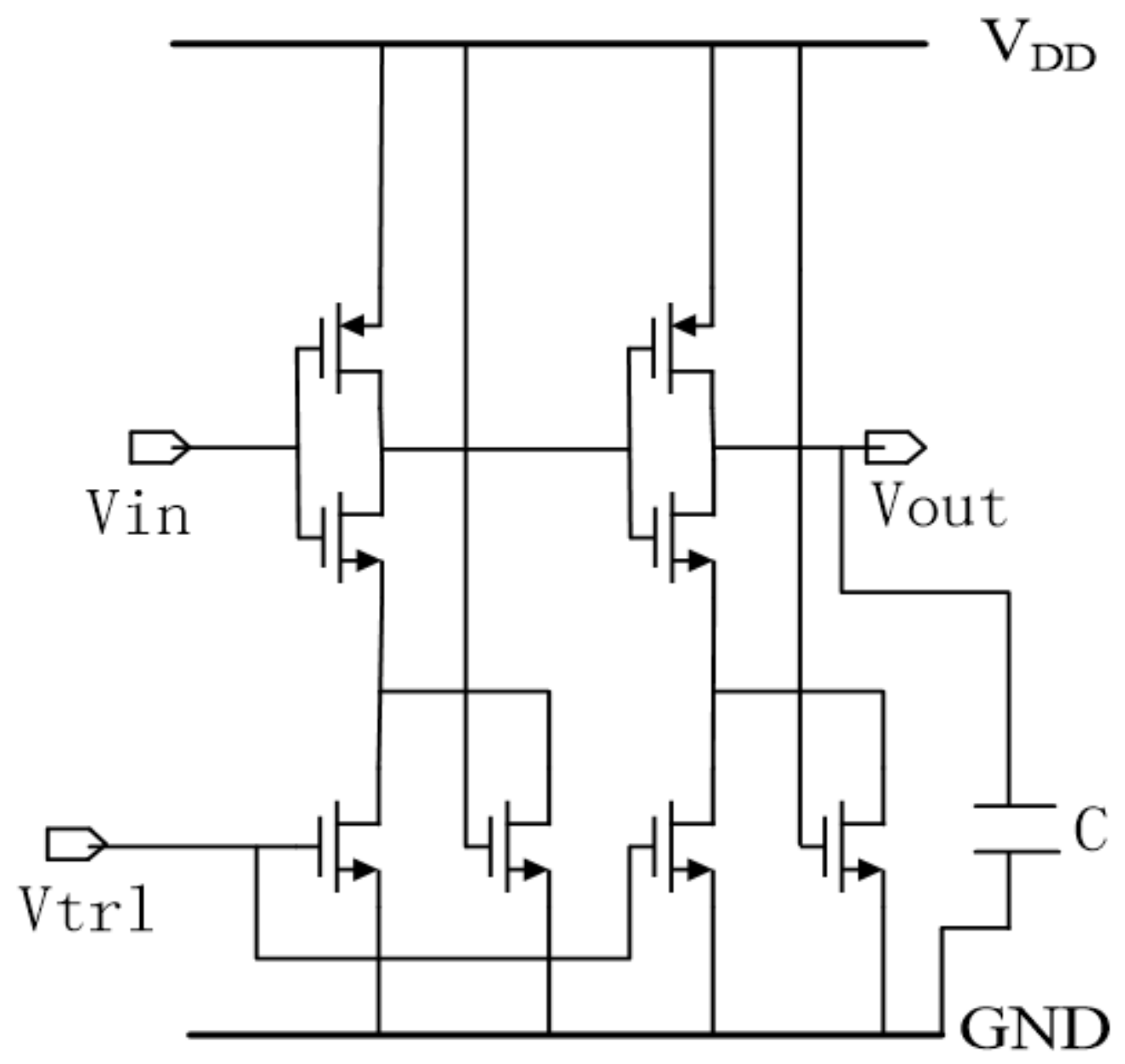

Figure 9

delay cell circuit 


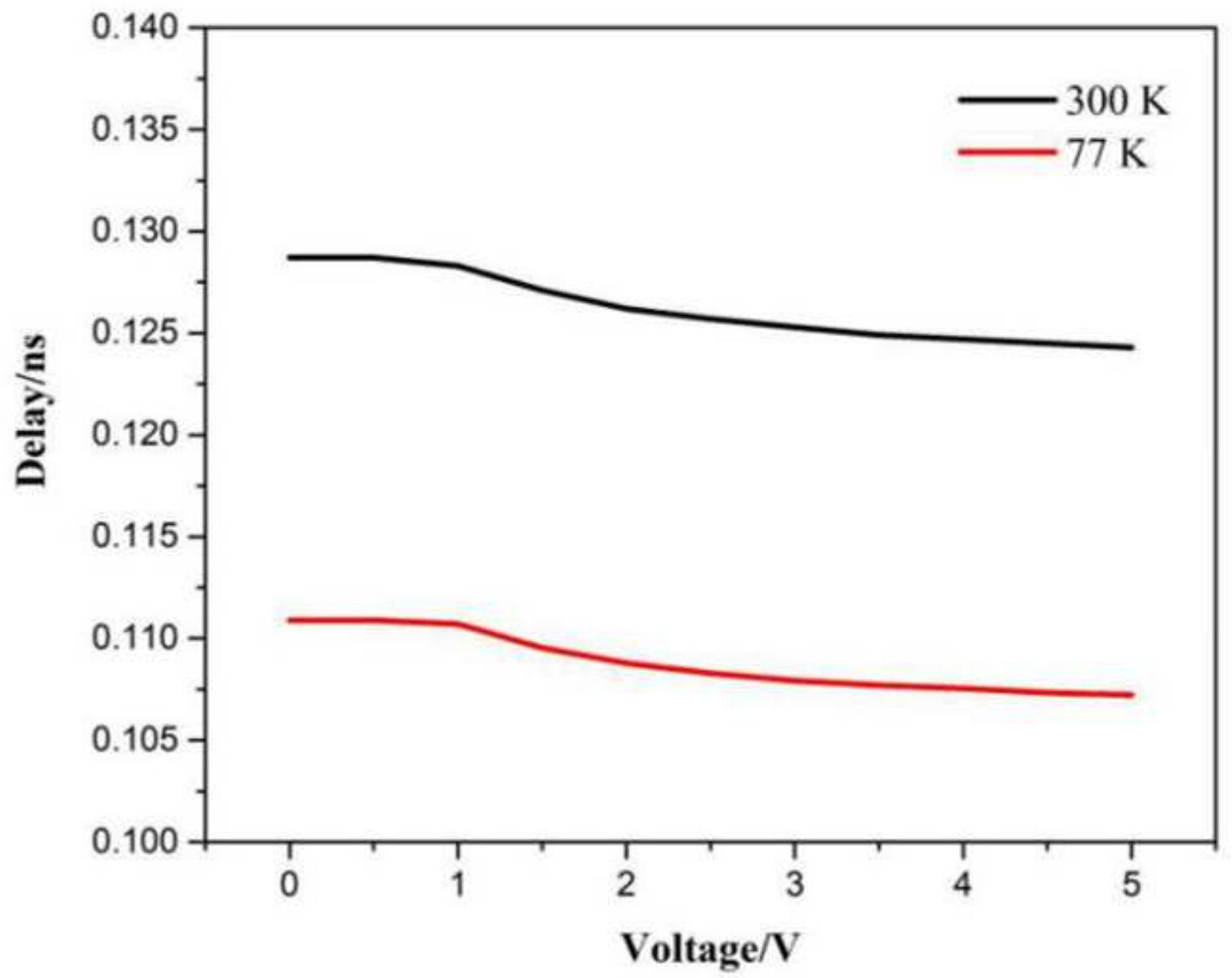

Figure 10

Delay time at different temperatures and different bias voltages 


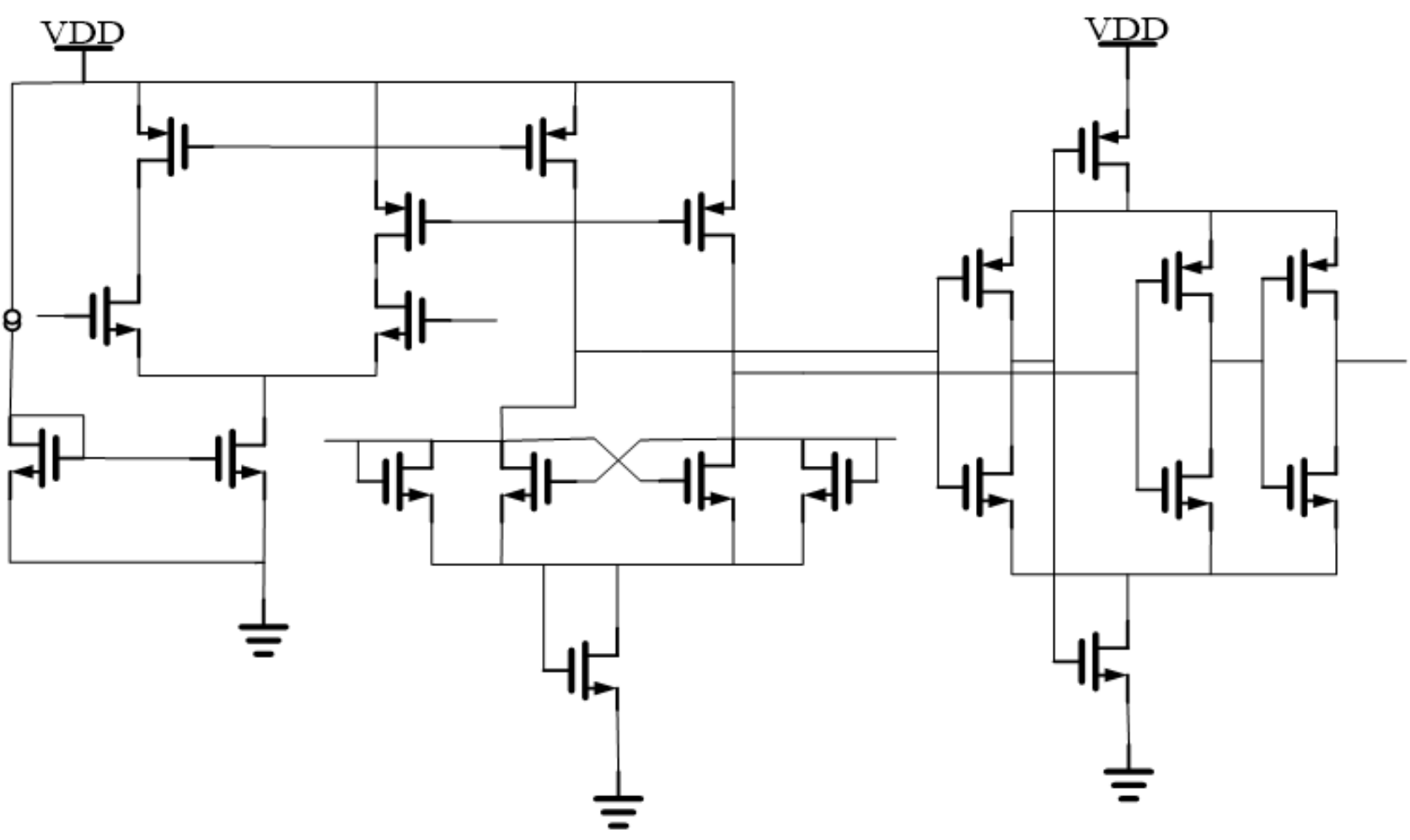

Figure 11

Comparator schematic diagram 


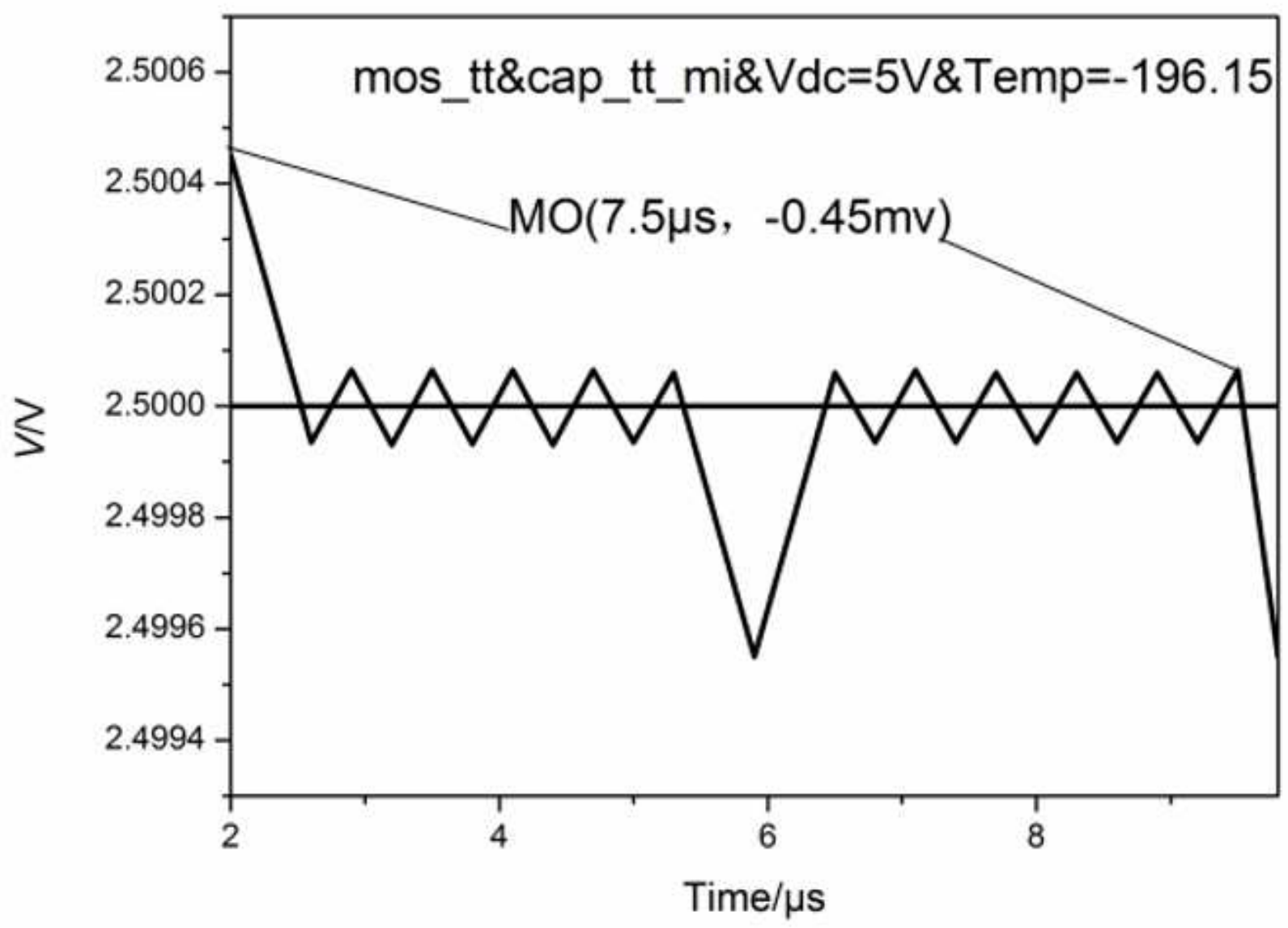

Figure 12

Simulation results of input offset voltage of comparator 


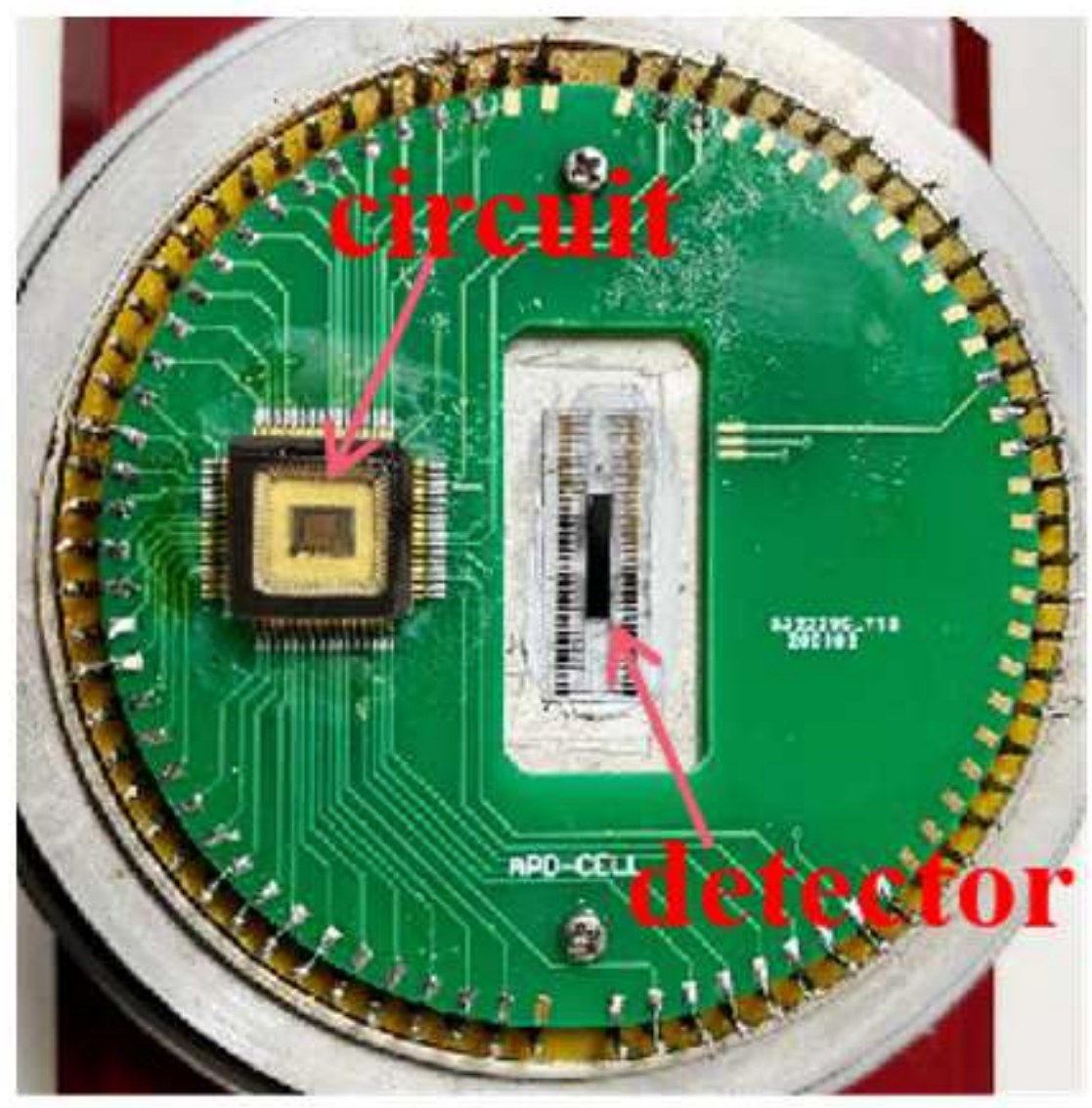

\section{Figure 13}

Tested chip in the Dewar

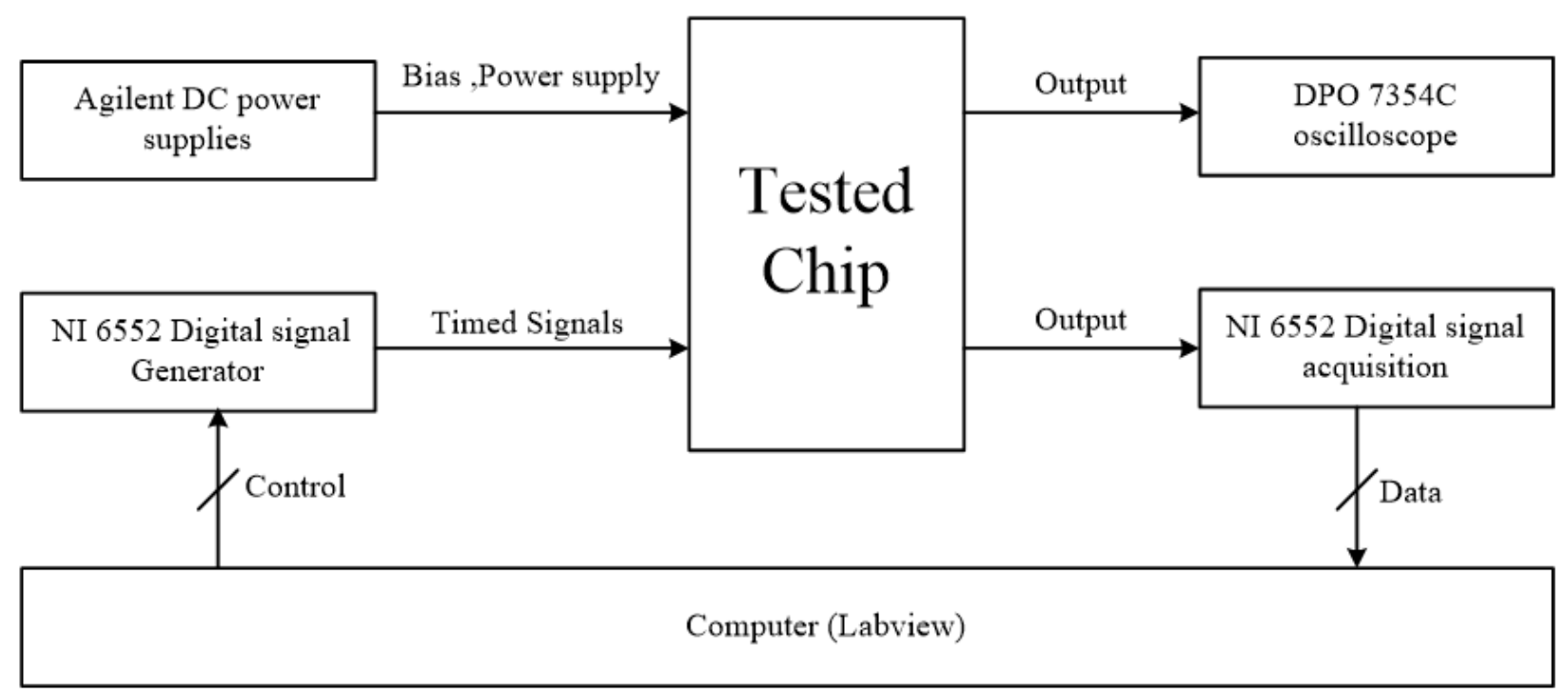

Figure 14 
Schematic block diagram of TDC circuit Testing system

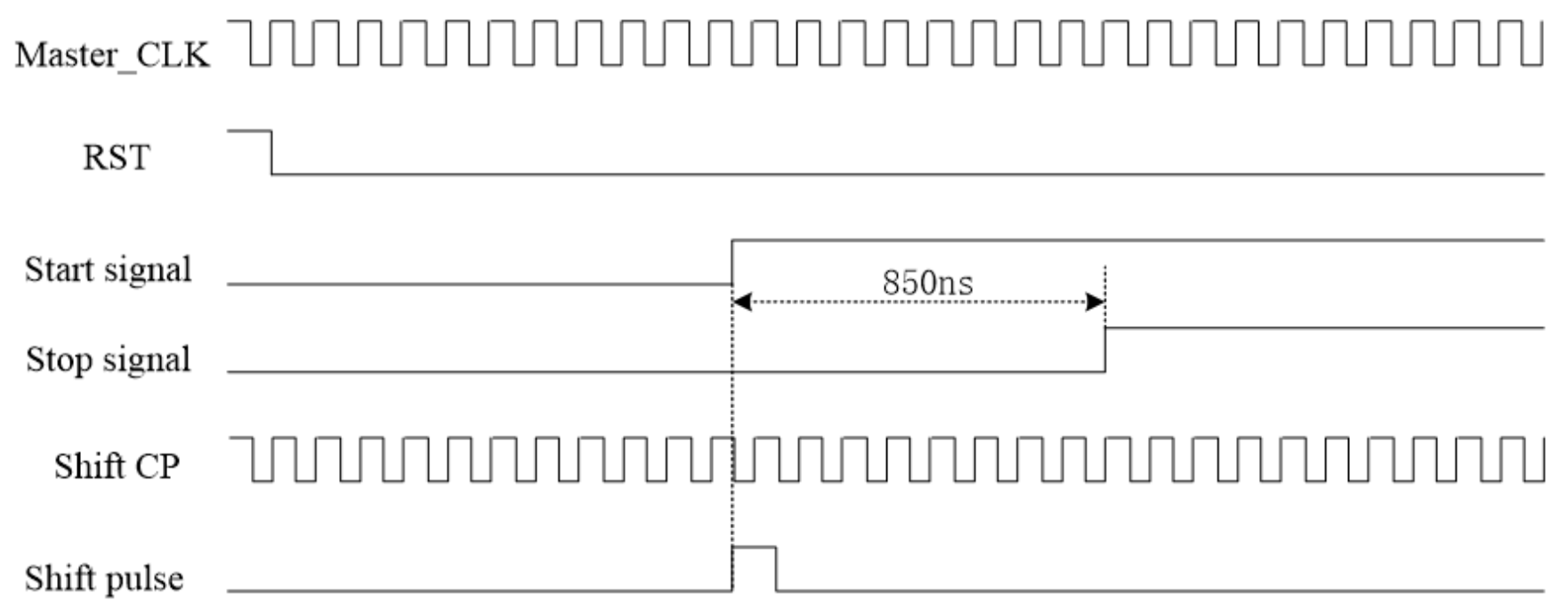

\section{Figure 15}

Test sequence diagram

Acquire data :Output

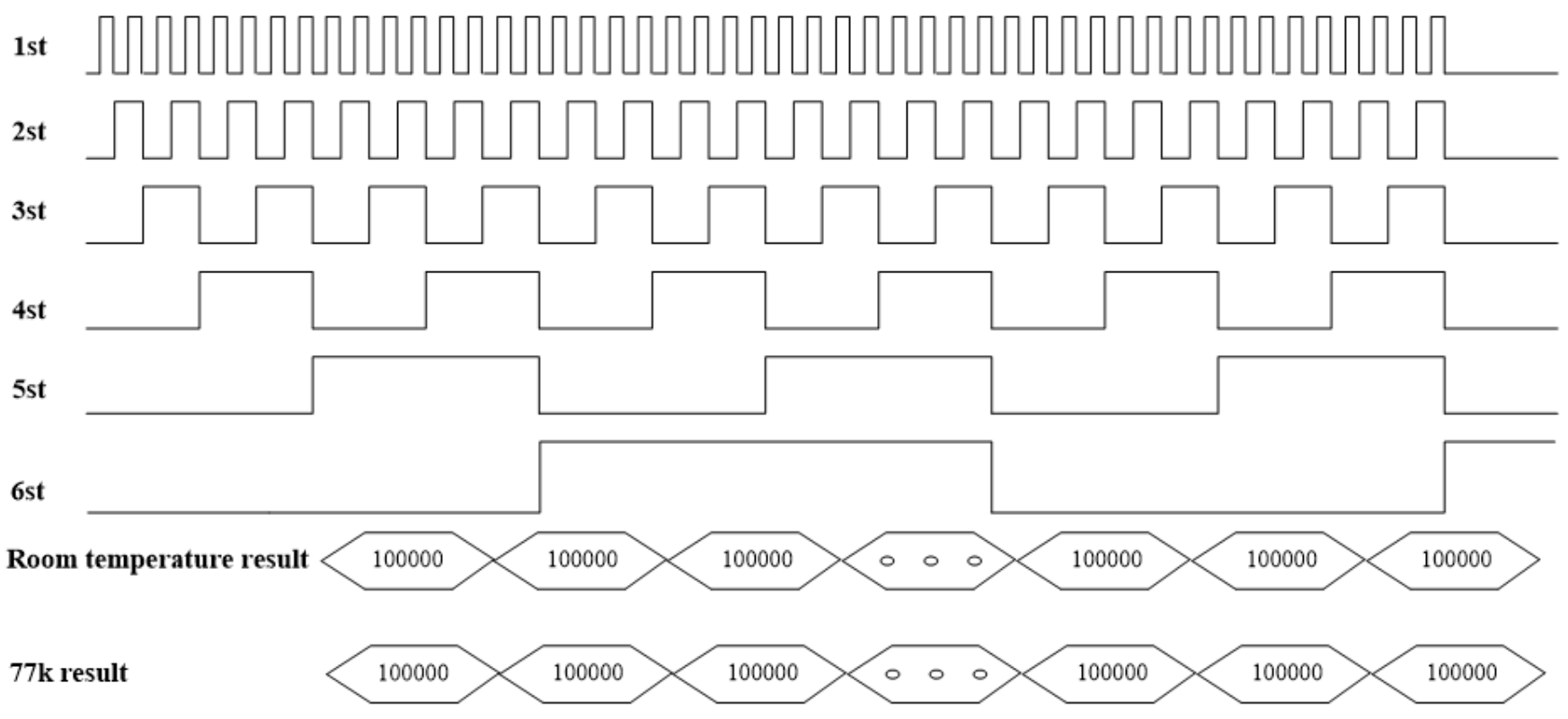

Figure 16

coarse counting test results at room temperature and low temperature 


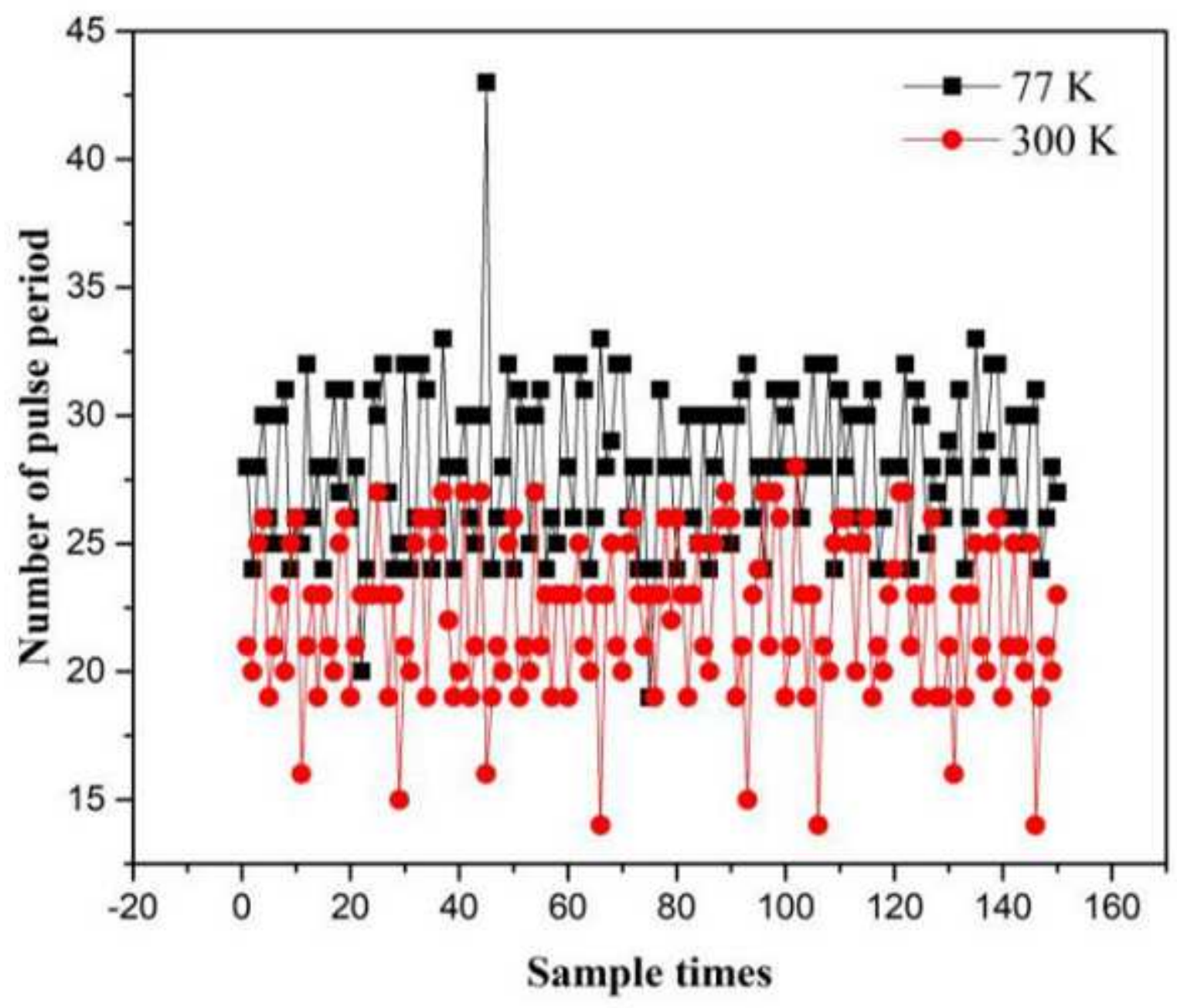

Figure 17

Fine counting value at room temperature and low temperature test 


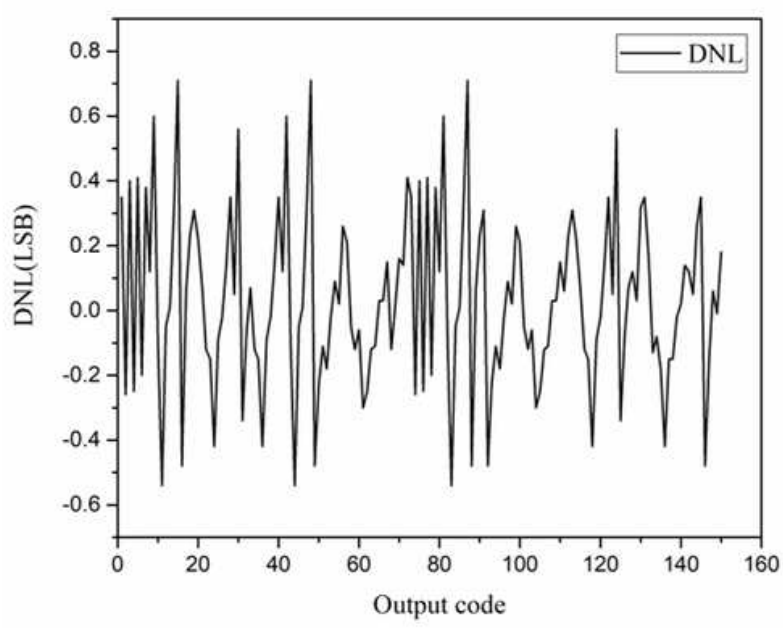

(a)

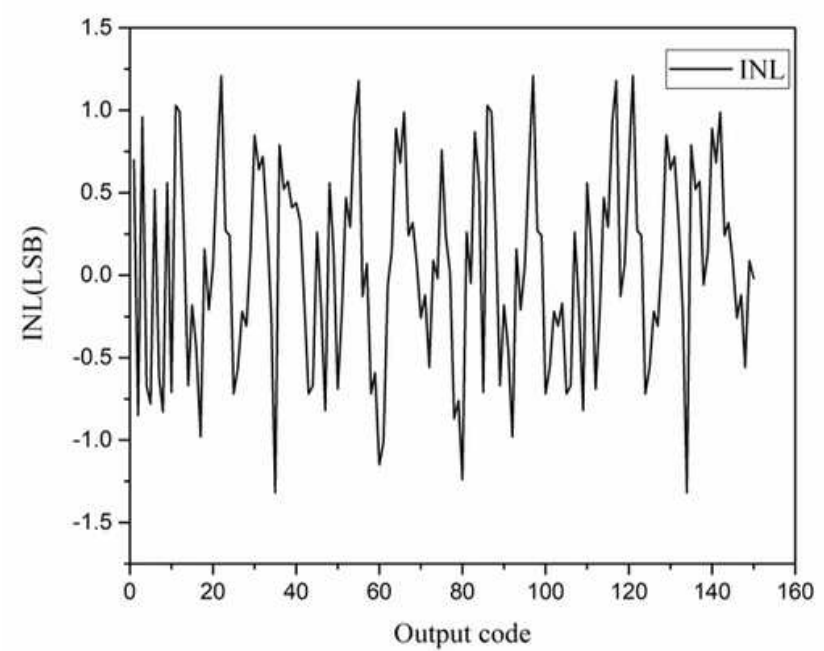

(b)

Figure 18

(a) Differential non-linearity;(b)Integral non-linearity 